\title{
On the Informed Seller Problem: Optimal INFORMATION Disclosure
}

\author{
Vasiliki Skreta ${ }^{*}$ \\ New York University, Stern School of Business
}

April 5, 2008

\begin{abstract}
We consider a revenue-maximizing seller who, before proposing a mechanism to sell her object(s), observes a vector of signals correlated with buyers' valuations. Each buyer knows only the signal that the seller observes about him, but not the signals she observes about other buyers. The seller first chooses how to disclose her information and then chooses a revenue-maximizing mechanism. We allow for very general disclosure policies, that can be random, public, private, or any mixture of these possibilities. Through the disclosure of information privately, the seller can create correlation in buyers' types, which then consist of valuations plus beliefs. For the standard independent private values model, we show that information revelation is irrelevant: irrespective of the disclosure policy an optimal mechanism for this informed seller generates expected revenue that is equal to her maximal revenue under full information disclosure. For more general allocation environments that allow also for interdependent, for common values, and for multiple items, disclosure policies may matter, and the best the seller can do is to disclose no information at all. Keywords: mechanism design, informed principal, information disclosure, correlated information, optimal auctions. JEL Classification Codes: C72, D44, D82.
\end{abstract}

\footnotetext{
${ }^{*}$ Leonard Stern School of Business, Kaufman Management Center, 44 West 4 th Street, KMC 7-64, New York, NY 10012,USA,vskreta@stern.nyu.edu.

${ }^{\dagger}$ I am grateful to an associate editor and three anonymous referees for numerous suggestions that led to a substantially improved paper. Many thanks to John Asker, Luis Cabral, Chris Edmond, Jan Eeckhaut, Philip Reny, Chris Phelan, Adam Szeidl, Balazs Szentes, Andrea Wilson and to William Zame for helpful conversations and comments. I am especially thankful to Narayana Kocherlakota for an illuminating discussion. Financial support from the National Science Foundation, Award \# 0451365 is gratefully acknowledged.
} 


\section{INTRODUCTION}

The literature on revenue-maximizing auctions ${ }^{1}$ is a cornerstone of auction theory. A crucial assumption in this literature is that the seller does not have any information that is unknown to the buyers. However, this assumption often fails in practice: A seller typically has superior information about how much objects are worth, and about participants' willingness to pay. In this paper, we consider a seller ${ }^{2}$ that has superior information about participants' willingness to pay. As in the work on revenue-maximizing auctions, the seller has the power to choose any auction to sell her objects. However, here we also allow the seller to disclose information to the buyers before the auction. We are interested in evaluating the impact of information disclosure on the revenue that the seller can generate, and in characterizing optimal information disclosure policies.

A striking example of a seller having information about buyers that is superior to the one their competitors have, is a sale of a company. A typical company sale includes a pre-auction stage where prospective buyers submit to the seller non-binding bids as an expression of interest, ("indicative bidding"). After indicative bidding, the seller knows a lot more about the various buyers' willingness to pay than their competitors do. Based on these indicative bids, the seller screens who should take part in the auction, ${ }^{3}$ and chooses a reservation price. Hansen (2001) suggests that these expressions of interest are close to how much bidders are actually willing to pay.

Similar phenomena occur in block trading. ${ }^{4}$ Prior to sale, institutional investors submit indications of interest, also known as IOI. IOI are very common and typically contain information about how many shares, and at what price, the particular buyer is willing to purchase. Another common instance where the seller may have information superior to the buyers' is sales of real estate. There, the seller typically observes how many times a buyer visits the house, how carefully he examines everything, whether or not he comes to the house with his/her architect, and, as in the case of company sales, preliminary statements about how much a buyer would be willing to pay at the auction. All this information is correlated with a buyer's willingness to pay. The seller may then know more about a buyer's competitors, than the buyer himself knows. The same can be true when sales (or purchases) are repeated. Consider, for instance, purchases by government agencies. In such situations, the government agency may have interacted in the past more often with some of the bidders and, thus, have information about their costs that is superior to that of the participants at the current auction. ${ }^{5}$

In this paper, we analyze the following problem. There is a risk-neutral seller who faces $I$

\footnotetext{
${ }^{1}$ The seminal papers are Myerson (1981) and Riley and Samuelson (1981).

${ }^{2}$ We use the female pronoun for the seller and male pronouns for the buyers.

${ }^{3}$ Given what we know from auction theory (see, for instance, Bulow and Klemperer (1996)) it may appear suboptimal for a seller to want to screen out buyers. For a justification, see Hansen (2001) or Milgrom (2004).

${ }^{4}$ Trade of a large number of shares, usually traded off the floor.

${ }^{5}$ However, we should mention that in practice, things may not be as simple as the description of the examples in the last two paragraphs suggests, because the seller and the entity organizing the auction could differ. Then, it is possible that the seller is controlling the pre-auction information disclosure phase, whereas an intermediary is choosing the auction rules. It is also possible that the intermediary does both. So long as the objectives both of the seller and the intermediary coincide and amount to revenue maximization, our analysis goes through.
} 
risk-neutral buyers whose willingness to pay depends on a single dimensional parameter $v_{i}$ that is private information, but is distributed according to $F_{i}$, which is common knowledge. Valuation parameters are independently distributed across buyers. The seller observes privately a vector of signals $s=\left(s_{1}, \ldots, s_{I}\right)$ one for each buyer. The vector of signals is taken to be exogenous ${ }^{6}$ and its components are distributed independently from one another. Each buyer knows only the signal that the seller has observed about him, but not the signals that the seller has observed about the others. Signal $s_{i}$ contains information about $v_{i}$ but not $v_{-i}$. At date 1 , the seller maps her information to one message for each buyer. We call this mapping information disclosure policy. We impose no restriction on what the seller can report, and we allow the seller to make private or public reports and to employ randomizations. At date 2, the seller proposes a revenue-maximizing mechanism to sell her object(s).

This is an informed principal problem with multiple agents, with the additional step that the principal (seller) can influence the agents' (buyers') perceptions about their competitors by disclosing information. How can a seller put her information to best use? Broadly speaking, the seller has three options: (1) no, (2) full, or, (3) some partial, possibly private, information disclosure. Each of these alternatives has very different implications for what buyers know when they are about to participate in the auction, which, in turn, may lead to big differences in achievable revenue. For example, when information disclosure is private, then, a buyer's beliefs about another buyer become part of his type since they depend on the information that is not publicly available, and buyers' types ${ }^{7}$ can become correlated, even though buyers' initial information was statistically independent. Can the seller create correlations that allow her to reduce the buyers' information rents? Can she eliminate the distortions of incomplete information by using mechanisms of the Cremer-McLean (1988) type? What is the optimal information disclosure policy, given that the seller will be subsequently choosing an optimal mechanism? We address these questions in the standard independent private value setup $(I P V)$, as well as in a general model that allows for interdependent values, for common values, and for multiple goods.

\footnotetext{
${ }^{6}$ In some of the examples mentioned earlier, indications of interest could be endogenous and could depend on what the seller plans to do with the information once it is released. However, it is important to note that in many situations, such as in company sales (which are one-time events), the information that the seller has about the possible auction participants often comes from previous interactions with such entities (they are suppliers, subcontractors, competitors) at points in time when the company sale was not anticipated. For those scenarios, which are quite common in sales of private companies, our assumption about the information being exogenous is probably more realistic than the reverse. The same is true in other situations, where the seller obtained information about various participants in the auctions, at points in time when the auction was not anticipated. For instance, in the case of real estate, the owner of a house may have heard some of her friends say how much they would be willing to pay for the house, at some point in time when the owner herself was not considering selling it.

Clearly, though, there are situations where the assumption that the seller's information is exogenous is less realistic. For these cases, our analysis is a partial-equilibrium one. With the given information in her hands, what is the best the seller can do? The results and the method of analysis, can be incorporated in larger games where the information released is endogenous, and it depends on what buyers anticipate that the seller will do in the future with the information they release.

7 "Types" then consist of valuations plus beliefs.
} 
What information disclosure policies are optimal is not clear a priori. A potential rationale for no information revelation is that more information makes more deviations for the buyers feasible, making, in some sense, the incentive constraints harder to satisfy. ${ }^{8}$ However, in quite a few instances, the reverse is true, and full information disclosure is the optimal policy. This is the case, for instance, in Milgrom and Weber (1982), who discover the celebrated "linkage principle," and in Eső and Szentes (2006). Also, the possibility of private information revelation may be strictly preferred to all public information disclosure policies. An environment where this can be true is analyzed by Harstad and Mares (2003).

We show that when the seller has the ability to use her information to design optimal mechanisms, the scope of information disclosure is limited. In the standard $I P V$ case, we have an Information Irrelevance Theorem: Irrespective of the disclosure policy, an optimal mechanism generates expected revenue that is equal to her revenue under full information disclosure. This is despite the fact that the seller can create correlation in buyers' types, ${ }^{9}$ and is in contrast to what happens with exogenously fixed auction rules, where the belief-relevant part of type has a significant impact on equilibrium behavior. This is demonstrated in Bergemann and Välimäki (2006). However, for general auction design problems, the seller's expected revenue can depend on which disclosure policy she employs. Then, the question that arises is which one is best. In response to this question, we show that the best information disclosure policy is to release no information at all. This finding may be somewhat surprising, given that we allow for interdependent values, which is also the case in Milgrom and Weber (1982), who prove the opposite result. However, there are two important differences between Milgrom and Weber (1982) and this paper. In Milgrom and Weber (1982), the auction rules are fixed, and the buyers' (prior) information is affiliated. Here, the seller can choose the auction she uses, and the prior information that buyers have is statistically independent.

There are three main forces behind our results. First, disclosure policies, irrespective of how sophisticated they are, essentially have no impact on a buyer's information rents. The reason for this is that a buyer can still "mimic" the behavior of the same set of valuations, as in the case where all the information that the seller has is public. Moreover, the expected payments that a seller can extract from "selling" buyers (agents) information about their competitors are always equal to zero. This is because there is a common prior and all players form posteriors using Bayes' rule, which implies that the side contracts written between the seller and a buyer that have positive expected value for the seller have negative expected value for the buyer. Thus, they are never accepted. The second force is that the seller's incentive constraints are not binding. This happens because the seller's information is non-exclusive: What she knows about a buyer, is also known to that particular buyer himself. The third force is related to how disclosure policies affect the set of incentive-compatible mechanisms. In the case of independent private values, disclosure policies do not enlarge the set of incentive-compatible mechanisms in any relevant way. The reason is

\footnotetext{
${ }^{8}$ Myerson (1986) studied an environment where this reasoning applies.

${ }^{9} \mathrm{~A}$ by-product of our analysis is that unless the seller observes information that tells her exactly what the valuation of a buyer is, full surplus extraction is not possible, irrespective of how sophisticated her disclosure policies are.
} 
that in the $I P V$ case, even when beliefs are part of buyers' types, an optimal Bayesian incentive compatible mechanism is also dominant strategy incentive compatible. This is not true in general, however. In general, disclosure policies affect the set of incentive-compatible mechanisms, which becomes largest when the seller discloses no information at all. This is why, in the general case, the best that the seller can do is to disclose no information at all. We now give a more comprehensive account of some related work.

\subsection{Related Literature}

This paper is related to the work on mechanism design by an informed principal. ${ }^{10}$ The first paper in this literature is Myerson (1983), which formulates the important idea of inscrutable mechanisms. Two other seminal contributions are Maskin and Tirole (1990) and (1992). Other than Myerson (1983), two papers allow for multiple agents: Tisljar (2003), under the restriction that buyers do not have private information, and Mylovanov (2005), which examines a private value quasilinear environment. In all the aforementioned papers, the principal's information pertains to his/her preferences and is exclusive. In this paper, the seller has information that is not commonly known, but each buyer knows the information that the seller has about himself; hence, the seller's information is non-exclusive.

Even though our informed seller problem (both in the baseline $I P V$ case, and in the general case) falls in the common value category, $C V_{\text {Informed_Principal }}{ }^{11}$ it has a private value, $P V_{\text {Informed_Principal }}{ }^{12}$ flavor in that the seller can always achieve her complete information ${ }^{13}$ payoff. ${ }^{14}$ Also, in our general model, the informed principal can do strictly better than an uninformed one, even though we are in a quasilinear setup. This is because the seller's private information relaxes the buyers' incentive constraints. This finding is in contrast to the analysis of Maskin and Tirole (1990) and Mylovanov (2005), who consider $P V_{\text {Informed_Principal }}$ environments and show that in the quasilinear case, the principal is indifferent between having private information and not. Finally, in our informed principal problem, the principal's information is correlated with the information of the agents. This is also the case in Cella (2007), who considers a single-agent, $P V_{\text {Informed_Principal, }}$ quasilinear environment, whereas, in this paper, the informed principal is sometimes strictly better off than an uninformed one. The forces present are different, however, since in our setup the asymmetric

\footnotetext{
${ }^{10}$ There are also papers that study the choices of an informed seller within specific classes of mechanisms. See, for instance Cai, Riley and Yi (2007) and Jullien and Mariotti (2006).

${ }^{11}$ In the terminology of Maskin and Tirole (1990) and (1992), in the common value case, the principal's private information enters in the agent's payoff function not only though the mechanism, but also in other ways, either directly or by affecting the agent's beliefs. In the case of private values, the principal's information enters only through the mechanism.

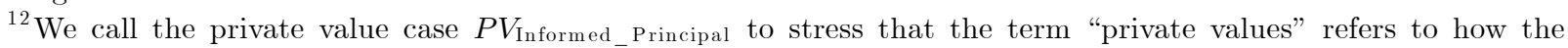
principal's information affects the agents' payoffs, and that this notion is completely distinct from the notion of the $I P V$ setting.

${ }^{13}$ Here, we follow Maskin and Tirole (1990) and call "complete information" the case where there is no uncertainty about the principal, even though the agent can have private information.

${ }^{14}$ This is because the principal can always achieve her complete information maximal payoff in a $P V_{\text {Informed_Principal }}$ environment, but not in a $C V_{\text {Informed_Principal }}$ environment (Maskin and Tirole (1992)).
} 
information between the buyers and the seller appears only if there is more than one buyer.

This paper is also related to the literature on mechanism design with endogenous types. ${ }^{15}$ In Obara (2008), agents' types are endogenous and are determined by actions agents choose before participating in the mechanism. He investigates under which circumstances the actions chosen generate an environment where the designer can extract full surplus from the agents. In the papers by Bergemann and Pesendorfer (2007), Eső and Szentes (2007), Ganuza (2004) and Ganuza and Penalva (2006), the endogeneity of information is directly controlled by the mechanism designer, or the seller. More specifically, the seller controls the technology that generates the value estimates for the buyers. Valuations are private, but in order for a buyer to assess how much the good is worth to him, he must receive information from the seller. Those papers consider such problems under different assumptions about the seller's technology to influence valuations. With the exception of Eső and Szentes (2007), ${ }^{16}$ in those papers, at an optimum, the seller discloses less information than is efficient: More information increases efficiency, which has a positive effect on revenue, but also increases information rents, which has a negative effect on revenue. Unlike these papers, in this paper, for the case of private values, buyers know their valuations, and the seller influences the belief-relevant part of their type. Another difference is that this paper also examines interdependent and common value environments. Moreover, here, the seller's information is private. Our question is not how the seller can best influence the buyers' own estimates in order to boost revenue, as is the focus of Bergemann and Pesendorfer (2007), Ganuza (2004), and Eső and Szentes (2007), but, rather, how the seller can influence a buyer's perceptions about his competitors in order to increase revenue.

Our findings are intimately related to the recent work on mechanism design with statistically correlated information by Neeman (2004), and by Heifetz and Neeman (2006). The classic papers in that literature (Cremer and McLean (1988), and McAfee and Reny (1992)) establish that when agents are risk-neutral, even an arbitrarily small correlation in types renders private information worthless and allows the designer to extract full surplus. In those papers, a type of an agent consists of a parameter that affects payoffs. In this paper, full surplus extraction is typically not possible, because, as in Neeman (2004) and Heifetz and Neeman (2006), beliefs are also part of the types. When this is the case, typically, there are many valuations that have the same beliefs; therefore, the knowledge of beliefs does not make the exact inference of valuations possible.

This work relates also to the large literature on information revelation in auctions. Perry and Reny (1999) show that the linkage principle of Milgrom and Weber (1982) may fail when there are multiple objects. Ottaviani and Prat (2001) show that the linkage principle holds in a non-

\footnotetext{
${ }^{15}$ For an excellent survey of this topic, as well as of other important topics about information in mechanism design, see Bergemann and Välimäki (2006).

${ }^{16}$ Eső and Szentes (2007) show that the inefficiency created by having too little information, can be eliminated by allowing the seller to charge a fee for the information she provides. In that paper, the seller simultaneously chooses the mechanism and how much information to disclose. Then, her incentives to disclose information are completely aligned with efficiency because her rents are tied to how big the pie will be. They establish that an optimal disclosure policy for the seller is to provide as much information as possible.
} 
linear pricing model where the monopolist first discloses information and then chooses an optimal schedule. Recently, Board (2006) allows for the possibility that information released changes the winner and shows that, in this case, the linkage principal fails when the number of buyers is two. With the exception of Ottaviani and Prat (2001), the aforementioned papers examine the effect of information disclosure for a given auction procedure. ${ }^{17}$ Also, by contrast with these papers, where buyers' information is affiliated, in this paper, the buyers' initial information is statistically independent.

Initial information is also statistically independent in Landsberger, Rubinstein, Zamir and Wolfstetter (2001), and in Kaplan and Zamir (2002). Landsberger, Rubinstein, Zamir and Wolfstetter (2001) show that when the seller knows the ranking of buyers' valuations and she makes it common knowledge, a first-price auction raises higher revenue than in the case where the seller does not disclose this information. Kaplan and Zamir (2002) also consider information revelation in firstprice auctions, but allow for more general public disclosure policies than Landsberger, Rubinstein, Zamir and Wolfstetter (2001) allow. There are three main differences between our paper and these. First, in Landsberger, Rubinstein, Zamir and Wolfstetter (2001) and Kaplan and Zamir (2002) the information that the seller receives is correlated across buyers. Because of this correlation, information disclosure creates a correlation in the payoff-relevant part of types, their valuations. Second, in those papers, information disclosure is public, whereas we allow the seller to employ public as well as, private disclosure policies. Finally, they consider first price auctions, whereas our seller designs revenue-maximizing mechanisms.

The remainder of this paper is structured as follows. Section 2 describes the environment and the main definitions used in the analysis. Section 3 contains the analysis of the information disclosure and mechanism selection problem in the standard independent private value case and establishes the information irrelevance theorem. Section 4 contains a brief description of the general model and establishes the no-disclosure result. We finish with a brief summary and some final remarks in Section 5. Proofs not found in the main text are in Appendix A.

\section{The Environment and Main Definitions}

A risk-neutral seller, indexed by 0 , owns a unit of an indivisible object, and faces $I$ risk-neutral buyers. The seller's valuation for the object is zero, whereas that of buyer $i$ is distributed on a set $V_{i}=\left[a_{i}, b_{i}\right]$ according to a continuous, and strictly positive density $f_{i}{ }^{18}$ A buyer's valuation $v_{i}$ is private and independently distributed across buyers. We use $f(v)=\times_{i \in I} f_{i}\left(v_{i}\right)$, where $v \in V=$ $\times_{i \in I} V_{i}$ and $f_{-i}\left(v_{-i}\right)=\times_{\substack{j \in I \\ j \neq i}} f_{j}\left(v_{j}\right)$.

The timing of the game is as follows:

\footnotetext{
${ }^{17}$ A somewhat intermediate approach is seen in Kremer and Skrzypacz (2004). There, the release of information from the seller is through which "standard" auction procedure she chooses.

${ }^{18}$ Our results go through when the priors $F_{i}$ are discrete or mixed measures. In such cases, the analysis can proceed essentially using techniques described in Skreta (2007). Further details are available from the author upon request.
} 
- At date 1 , the seller observes privately a vector of signals $s=\left(s_{1}, \ldots, s_{I}\right)$ and maps it (possibly randomly) to a vector of messages $m=\left(m_{1}, \ldots, m_{I}\right)$, one for each buyer.

- At date 2, the seller chooses an auction procedure to sell the object. Date 2 has three stages. At stage one, the mechanism is proposed and at stage two, the buyers accept or reject the mechanism. If they all accept, the mechanism is played at stage three; otherwise, buyers get their outside option, which is 0 , and the seller keeps the good.

Date 1 captures some previous interaction between the seller and the buyers, such as a previous auction, or some other process, such as the indicative bidding stage in the sales of private companies or the IOIs in block trading. On Date 2, the auction procedure is chosen and carried out. We now proceed to give a more detailed account of the seller's information, how she maps it to messages to the buyers, and the effect of this information disclosure on the buyers' and the seller's types.

Seller's Information: The vector of signals $s$ is taken to be exogenous. We use $S_{i}$ to denote the set of signals that the seller can observe about buyer $i: S \equiv \times_{i \in I} S_{i}$ to denote the set of vectors of signals about all buyers, and $S_{-i}=\times_{i \in I} S_{i}$ to denote the set of vectors of signals about all buyers, but $i$. For simplicity, we take the set $S_{i}$ to be finite. No result, however, hinges on this simplification. Let $\pi_{i}\left(s_{i}\right)$ denote the probability that the seller observes signal $s_{i}$ about $i, i \in I$. These probabilities are common knowledge. We assume that $s_{i}$ 's are independently distributed from one another and use $\pi(s)=\times_{i \in I} \pi_{i}\left(s_{i}\right)$ to denote the joint distribution. Each signal $s_{i}$ is potentially informative about buyer $i$ 's valuation, $v_{i}$, but it does not contain any information about $v_{j}$ for $j \neq i$. The posterior of $v_{i}$ conditional on $s_{i}$ is denoted by $f_{i}\left(v_{i} \mid s_{i}\right)$, which is assumed to be strictly positive and continuous on $V_{i}\left(s_{i}\right)=\left[\underline{v}_{i}\left(s_{i}\right), \bar{v}_{i}\left(s_{i}\right)\right]$, where $\underline{v}_{i}\left(s_{i}\right)$ and $\bar{v}_{i}\left(s_{i}\right)$ are respectively the lowest and highest valuations in the support of $f_{i}\left(v_{i} \mid s_{i}\right) .{ }^{19}$ Each buyer knows only the signal the seller has observed about himself $\left(s_{i}\right)$, but not the ones that the seller has observed about the other buyers, the $s_{-i}$ 's. For our results here, as well as in the more general model considered later, it does not make any difference whether the seller's information is verifiable or not.

Information Disclosure: The part of the seller's strategy that specifies the relationship between what she knows and what she reports to the buyers is called an information disclosure policy. More formally, an information disclosure policy is a mapping from the vector of signals observed by the seller to a vector of messages, one for each buyer, that is, $c: S \rightarrow \Delta(\mathcal{M})$, where $\mathcal{M}:=\times_{i \in I} \mathcal{M}_{i}$, and where $\mathcal{M}_{i}$ is the set of messages that the seller can send to buyer $i$. The vector of messages revealed is $m=\left(m_{1}, m_{2}, \ldots, m_{I}\right)$, with $m_{i} \in \mathcal{M}_{i}, i \in I$. With a slight abuse of notation, we use $c(m \mid s)$ to denote the probability that the disclosure policy reveals $m$ given $s$. The disclosure policy is common knowledge, but each buyer observes only $m_{i} \cdot{ }^{20}$

\footnotetext{
${ }^{19}$ This assumption is not essential. Our results go through when the posteriors $F_{i}\left(. \mid s_{i}\right)$ are discrete or mixed measures. Further details are available from the author upon request.

${ }^{20}$ At an equilibrium, this map is understood by all. Alternatively, we could have the seller choose the mapping $c$ before she sees $s$. For our analysis, this difference turns out to be immaterial.
} 
Types: A type of a player consists of whatever he knows, that is not common knowledge. Here, both the buyers and the seller have private information. Buyer $i$ 's beliefs about $j$ are determined from the prior and the disclosure policy conditional on $s_{i}$ and $m_{i}$. Because $s_{i}$ and $m_{i}$ are privately observed, $i$ 's beliefs about $-i$ are not known to the other buyers and they become part of buyer $i$ 's type. Hence, buyer $i$ 's type consists of a payoff-relevant part, $v_{i}$, and a belief-relevant part, $s_{i}, m_{i}{ }^{21}$ The set of types of buyer $i$ is denoted by $V_{i}\left(S_{i}\right) \times S_{i} \times \mathcal{M}_{i}\left(S_{i}\right)$, and it consists of triplets of the form $v_{i}, s_{i}, m_{i}$, where $v_{i} \in V_{i}\left(s_{i}\right)$ and $m_{i} \in \mathcal{M}_{i}\left(s_{i}\right)$, for $s_{i} \in S_{i}$, where a message $m_{i}$ is in $\mathcal{M}_{i}\left(s_{i}\right)$ if $c\left(m_{i}, \tilde{m}_{-i} \mid s_{i}, \tilde{s}_{-i}\right)>0$, for some $\tilde{s}_{-i} \in S_{-i}$ and $\tilde{m}_{-i} \in \mathcal{M}_{-i}{ }^{22}$ The set of types for the seller is denoted by $S \times \mathcal{M}(S)$, and it consists of vectors $(s, m)$, where $s \in S$ and $m \in \mathcal{M}(s)$, and where the set $\mathcal{M}(s)$ contains all messages that satisfy $c(m \mid s)>0$. A type profile is given by

$$
(\underbrace{v_{i}, s_{i}, m_{i}}_{\text {buyer } i} ; \underbrace{v_{-i}, s_{-i}, m_{-i}}_{\text {buyers }-i} ; \underbrace{s_{i}, s_{-i}, m_{i}, m_{-i}}_{\text {seller }})
$$

which for convenience is summarized as $(v, s, m)$. The set of all type profiles is denoted by $V(S) \times$ $S \times \mathcal{M}(S)$.

Information Disclosure Policies \& Types - Examples: In the case of a disclosure policy that is fully revealing, everyone has observed the same amount of information, and the seller's and buyer $i$ 's beliefs about buyers $-i$ coincide and are common knowledge. In this case, buyer $i$ 's type consists merely of $v_{i}$. In the case of no information revelation, buyer $i$ 's posterior about $-i$ is equal to the prior. In that case, $i$ 's type is the couple $v_{i}, s_{i}$, since both $v_{i}$ and $s_{i}$ are known to $i$ but not to other buyers. For other disclosure policies, $i$ 's type is the triplet $v_{i}, s_{i}, m_{i}$.

Disclosure POLICY

fully revealing: $m_{i}=s$, for all $i$ and $s \in S$

no information revelation: $m_{i}=\emptyset$, for all $i$

"partial" information revelation: $m_{i}=\left\{\begin{array}{l}s_{-i} \text { w. pr } 0.5 \\ \hat{s}_{-i} \text { w. pr } 0.5\end{array}\right.$
TYPE OF BUYER $i$

$v_{i}$

$v_{i}, s_{i}$

$v_{i}, s_{i}, m_{i}$

Mechanism Selection Stage: Our seller here is informed, so formally, the problem of mechanism selection at date 2 belongs in the class of informed principal problems and is modeled as a three-stage game between a principal (seller) and the agents (buyers).$^{23}$ At stage 3 , the beliefs of the agents are updated to account for what they infer from the mechanism that the principal proposed at stage one. Also, the principal updates her beliefs about the agents' types after observing

\footnotetext{
${ }^{21}$ Note that $s_{i}$ is part of $i^{\prime} s$ type because it is known to $i$, but not to $-i$.

${ }^{22}$ The set $\mathcal{M}_{i}\left(s_{i}\right)$ contains all the messages that buyer $i$ receives with strictly positive probability, when the signal that the seller has observed about him is $s_{i}$.

${ }^{23}$ See Maskin and Tirole (1990) and (1992).
} 
that they all participated. Obviously, this kind of game can have a plethora of equilibria. Moreover, its analysis can be quite challenging. We follow an indirect approach, which is possible because of the beautiful insights from the path-breaking papers of Myerson (1983) and Maskin and Tirole (1990 and 1992). Once we are done with our somewhat "reduced form analysis," we argue why the allocations we obtain correspond to equilibrium allocations of the three-stage game. However, we will not be very explicit about all the details that are similar to the ones in the previous literature.

Let's start by abstracting, for the moment, from who has the power to choose the mechanism. By the revelation principle ${ }^{24}$ we can obtain all the equilibrium-feasible allocations arising from arbitrary games, by looking at equilibrium allocations of direct revelation mechanisms, that satisfy incentive, $I C$, participation, $P C$, and resource constraints, $R E S$, for all buyers and the seller. As usual, the seller and buyers send their reports simultaneously in the mechanism.

A direct revelation mechanism, $(D R M), M=(p, x)$ consists of an assignment rule $p: V(S) \times$ $S \times \mathcal{M}(S) \longrightarrow \Delta(I)$ and a payment rule $x: V(S) \times S \times \mathcal{M}(S) \longrightarrow \mathbb{R}^{I}$. The assignment rule specifies a probability distribution over the set of buyers given a vector of reports. We denote by $p_{i}(v, s, m)$ the probability that $i$ obtains the good when the vector of reports is $v, s, m$. Similarly, $x_{i}(v, s, m)$ denotes the expected payment incurred by $i$, given $v, s, m$.

What makes an informed principal problem special, is precisely the fact that the person in charge of choosing $(p, x)$ has private information. One consequence of that is that the seller must be also submitting reports in the mechanism. However, the most important implication, is that the choice of the schedule $(p, x)$ itself can reveal information to the buyers about the seller's information. This can happen because different types of the seller may prefer different $(p, x)$ 's. ${ }^{25}$ This information release changes the buyers' beliefs, and in order for $(p, x)$ to be feasible, it must satisfy the incentive and the participation constraints of the buyers with respect to their posterior beliefs, obtained after they see the mechanism chosen by the seller. The inscrutability principle due to Myerson (1983) argues that it is without any loss to restrict attention to incentive-feasible allocations, where all types of the individual in charge of choosing the mechanism, (the seller in our case), choose the same schedule. Then, the choice of the schedule itself does not reveal any additional information.

Fix an information disclosure policy $c$ and consider a DRM $p$ and $x$, (revelation principle). By the inscrutability principle, all types of the seller choose the same $(p, x)$, which implies that after being confronted with $(p, x)$, the buyers' beliefs about the seller's type do not change (the mechanism $(p, x)$ does not release any new information). Let

$$
u_{i}(v, s, m)=p_{i}(v, s, m) v_{i}-x_{i}(v, s, m),
$$

denote buyer $i$ 's payoff given a mechanism $(p, x)$ when the realized types are summarized to $v, s, m$, and all report truthfully. We let $P_{i}\left(v_{i}, s_{i}, m_{i}\right)=E_{v_{-i}, s_{-i}, m_{-i}}\left[p_{i}(v, s, m) \mid s_{i}, m_{i}\right]$ denote the expected probability that $i$ obtains the good, and $X_{i}\left(v_{i}, s_{i}, m_{i}\right)=E_{v_{-i}, s_{-i}, m_{-i}}\left[x_{i}(v, s, m) \mid s_{i}, m_{i}\right]$ denote the

\footnotetext{
${ }^{24}$ See, for instance, Myerson (1979) or Myerson (1981).

${ }^{25}$ Related to this point, Yilankaya (1999) shows in a bilateral trade setting that (holding the beliefs of the buyer constant) some types of the seller prefer a double auction, whereas other types prefer a posted price. At an optimum, however, the buyer understands which types of the seller prefer the double auction, which overturns its optimality.
} 
expected payment that he incurs, when his type is $v_{i}, s_{i}, m_{i}$ and when all other buyers and the seller report truthfully. These expectations are conditioned only on $s_{i}$ and $m_{i}$, and not on $v_{i}$ because valuations are independently distributed, which immediately implies that $v_{i}$ does not contain any information about $v_{-i}$. Also, since the disclosure policy maps $S$ to $\mathcal{M}, v_{i}$ does not contain any additional information for $m_{-i}$ and $s_{-i}$. The expected payoff of type $v_{i}, s_{i}, m_{i}$ of buyer $i$ at a truth-telling equilibrium of a direct revelation mechanism is given by

$$
U_{i}\left(v_{i}, s_{i}, m_{i}\right) \equiv E_{v_{-i}, s_{-i}, m_{-i}}\left[u_{i}(v, s, m) \mid s_{i}, m_{i}\right]=P_{i}\left(v_{i}, s_{i}, m_{i}\right) v_{i}-X_{i}\left(v_{i}, s_{i}, m_{i}\right) .
$$

A mechanism is feasible if and only if it satisfies the following incentive $(I C)$, participation $(P C)$ and resource $(R E S)$ constraints. The constraints for the seller are given by

$\mathbf{I C}_{0}: \int_{V(s)} \Sigma_{i \in I} \Sigma_{m \in \mathcal{M}} c(m \mid s) x_{i}(v, s, m) f(v \mid s) d v \geq \int_{V(s)} \Sigma_{i \in I} \Sigma_{m \in \mathcal{M}} c(m \mid s) x_{i}(v, \hat{s}, \hat{m}) f(v \mid s) d v$ for all $(s, m) ;(\hat{s}, \hat{m}) \in S \times \mathcal{M}(S)$ $\mathcal{M}(S)$.

$\mathbf{P C}_{0}: \int_{V(s)} \Sigma_{i \in I} \Sigma_{m \in \mathcal{M}} c(m \mid s) x_{i}(v, s, m) f(v \mid s) d v \geq 0$, for all $(s, m) ;(\hat{s}, \hat{m}) \in S \times$

The constraints for each buyer $i$, with $i \in I$, are given by:

$\mathbf{I C}_{i}: U_{i}\left(v_{i}, s_{i}, m_{i}\right) \geq P_{i}\left(\hat{v}_{i}, \hat{s}_{i}, \hat{m}_{i}\right) v_{i}-X_{i}\left(\hat{v}_{i}, \hat{s}_{i}, \hat{m}_{i}\right)$, for all $\left(v_{i}, s_{i}, m_{i}\right) ;\left(\hat{v}_{i}, \hat{s}_{i}, \hat{m}_{i}\right) \in$ $V_{i}\left(S_{i}\right) \times S_{i} \times \mathcal{M}_{i}\left(S_{i}\right)$.

$\mathbf{P C}_{i}: U_{i}\left(v_{i}, s_{i}, m_{i}\right) \geq 0$, for all $\left(v_{i}, s_{i}, m_{i}\right) \in V_{i}\left(S_{i}\right) \times S_{i} \times \mathcal{M}_{i}\left(S_{i}\right)$.

Resource Constraints are given by

RES : $p_{i}(v, s, m) \in[0,1]$ and $\Sigma_{i \in I} p_{i}(v, s, m) \leq 1$, for all $(v, s, m) \in V(S) \times S \times \mathcal{M}(S)$.

Before we proceed to investigate the consequences of these constraints, a few remarks are in order. In terms of the seller's incentive constraints, first note that, because we are analyzing mechanism selection after the information disclosure stage has taken place, the seller's reports enter only the mechanism and not the disclosure policy. This is because, at the point where the mechanism is chosen, the seller has already observed $s$ and disclosed information according to $c$. More importantly, since the seller's information is non-exclusive any deviations from the seller can be immediately detected when all the buyers report truthfully. However, since we do not a priori know from which side it is easier to elicit the non-exclusive information, namely the $s, m$, we write the constraints as if all information is exclusive. This implies that we also allow each buyer to misreport about all components of his type. Obviously, when $i$ lies about $s_{i}$ or about $m_{i}$, the seller can immediately detect this; nevertheless, these are possible deviations and we include them for completeness. Notice, however, that we do not allow the buyers and the seller to make reports that are incompatible with $c$. It is easy to see that such kinds of misreportings are easily detectable and, thus, immaterial. 
Summarizing, we are considering a problem where the mechanism designer has private information that is correlated with buyers' willingness to pay. This information can be used to affect buyers' types by affecting their beliefs about each other. Types then become correlated, since different types of a buyer have different beliefs about the types of the other buyers. Our objective is to evaluate the role of information disclosure on the performance of revenue-maximizing mechanisms that are chosen at date 2 . The next section contains the analysis of this problem in the standard $I P V$ case.

\section{Optimal Information Disclosure \& Mechanism Selection in the Standard IPV Case: An Information Irrelevance Theorem}

In this section we analyze the information disclosure and mechanism selection problem in the standard independent private value case and establish the information irrelevance theorem. Before we begin the general analysis of the problem, we present an example that illustrates the main forces behind the information irrelevance result.

\subsection{A Motivating Example}

Suppose that there two bidders, whose valuations are drawn from the uniform distribution on $[0,1]$. The seller can either observe a low $(L)$ or a high $(H)$ signal about each buyer. If a buyer's valuation falls in $[0,0.5]$, the seller observes $L$; otherwise, she observes $H$. Then, her posterior after observing $L$ for a buyer is that this buyer's valuation is uniformly distributed on $[0,0.5]$, and her corresponding posterior after observing $H$ is the uniform distribution on $[0.5,1]$.

Revenue-Maximizing Mechanisms under Full Disclosure: If the seller discloses all her information fully and truthfully, then a buyer's and the seller's beliefs about the other buyer coincide and are common knowledge. Suppose, for instance, that the seller has observed $L$ for both buyers, that is, $s_{1}=L$ and $s_{2}=L$. Then, when the seller employs the full disclosure policy, she sends $m_{1}=L$ and $m_{2}=L$ with probability one to each buyer. Using standard arguments (see Myerson (1981)) the seller's expected revenue when $s_{1}=L$ and $s_{2}=L$ can be written as

$$
\begin{aligned}
& \int_{0}^{0.5} \int_{0}^{0.5}\left[p_{1}\left(v_{1}, v_{2}, L, L, L, L\right)\left(2 v_{1}-0.5\right)+p_{2}\left(v_{1}, v_{2}, L, L, L, L\right)\left(2 v_{2}-0.5\right)\right] \frac{1}{0.5} d v_{1} \frac{1}{0.5} d v_{2} \\
& -\underline{U}_{1}(0)-\underline{U}_{2}(0),
\end{aligned}
$$

where $\underline{U}_{i}$ stands for the expected payoff of buyer $i$ when his valuation is equal to its lowest possible value. As is well known, the best the seller can do is to assign the object with probability one to the buyer with the highest virtual valuation, so long as his valuation is above 0.25 , and to keep the object otherwise. This assignment rule guarantees that the last two terms are identically equal to zero. Similarly, when the seller observes $s_{1}=H$ and $s_{2}=H$ her expected revenue can be written 
as

$$
\begin{aligned}
& \int_{0.5}^{1} \int_{0.5}^{1}\left[p_{1}\left(v_{1}, v_{2}, H, H, H, H\right)\left(2 v_{1}-1\right)+p_{2}\left(v_{1}, v_{2}, H, H, H, H\right)\left(2 v_{2}-1\right)\right] \frac{1}{0.5} d v_{1} \frac{1}{0.5} d v_{2} \\
& -\underline{U}_{1}(0.5)-\underline{U}_{2}(0.5) .
\end{aligned}
$$

In this case, the best for the seller is to assign the object with probability one to the buyer with the highest valuation, so long as his valuation is above 0.75 , and to keep the object otherwise. Finally, when the seller observes a high signal for one of the buyers and a low signal for the other one - say, for instance, $s_{1}=H$ and $s_{2}=L$ - then the seller's expected revenue can be written as

$$
\begin{aligned}
& \int_{0}^{0.5} \int_{0.5}^{1}\left[p_{1}\left(v_{1}, v_{2}, H, L, L, H\right)\left(2 v_{1}-1\right)+p_{2}\left(v_{1}, v_{2}, H, L, L, H\right)\left(2 v_{2}-0.5\right)\right] \frac{1}{0.5} d v_{1} \frac{1}{0.5} d v_{2} \\
& -\underline{U}_{1}(0.5)-\underline{U}_{2}(0) .
\end{aligned}
$$

In this case the best for the seller is to assign the object with probability one to buyer 1 if $v_{1} \geq$ $v_{2}+0.5$, to buyer 2 if $v_{2} \geq v_{1}-0.5$ and $v_{2} \geq 0.25$, and to keep the object otherwise. By exchanging 1 and 2 , we get the optimal auction outcome when the seller observes the low signal for buyer 1 and the high signal for buyer 2 .

The assignment rule we described is the best that the seller can achieve given full disclosure because it maximizes her revenue in all possible scenarios, $(L L, H H, H L$ and $L H)$. We now argue that this schedule is feasible under full disclosure and, hence, is a solution to the seller's problem. Actually, we argue something stronger, namely that it is feasible for all disclosure policies. First, it is easy to see (following exactly the arguments in Myerson (1981)) that this schedule is dominant strategy incentive compatible and it satisfies voluntary participation constraints for the buyers irrespective of their beliefs, which implies that it satisfies the constraints for the buyers for all disclosure policies. Second, the schedule is feasible for the seller - if the seller misreports, it would be as if she were picking a $p_{1}$ and $p_{2}$ that maximize a wrong objective function. Also, by construction, this allocation rule guarantees the seller a payoff that is greater or equal to her value, hence satisfying the seller's participation constraints. Since the seller's objective function depends only on $s$ and not on anything that can be affected by the disclosure policy, the same arguments show that the described allocation rule satisfies the seller's constraints irrespective of the disclosure policy. The fact that the optimal mechanism under full disclosure is feasible for all disclosure policies (together with the reasons behind this result that we just explained) is one of the main forces of our information irrelevance theorem.

Revenue-Maximizing Mechanisms under Alternative Disclosure Policies: Suppose, that the seller employs a disclosure policy other than full information disclosure. Then, the seller's and buyer $i$ 's beliefs about $j$ 's valuation differ, and the standard arguments used in obtaining an expression of the seller's revenue as a function of the allocation rule have to be modified. Below, we show that for disclosure policies $c$, other than the full disclosure policy, the seller's revenue when 
she has observed signals, say $s=(L, L)$, and has released messages $\left(m_{1}, m_{2}\right)$ is given by

$$
\begin{aligned}
& \int_{0}^{0.5} \int_{0}^{0.5}\left[p_{1}\left(v_{1}, v_{2}, L, L, m_{1}, m_{2}\right)\left(2 v_{1}-0.5\right)+p_{2}\left(v_{1}, v_{2}, L, L, m_{1}, m_{2}\right)\left(2 v_{2}-0.5\right)\right] \frac{1}{0.5} d v_{1} \frac{1}{0.5} d v_{2} \\
& -\underline{U}_{1}\left(0, L, L, m_{1}, m_{2}\right)-\underline{U}_{2}\left(0, L, L, m_{1}, m_{2}\right) \\
& +\int_{0}^{0.5} \int_{0}^{0.5}\left[\tau_{1}\left(v, L, L, m_{1}, m_{2}\right)+\tau_{2}\left(v, L, L, m_{1}, m_{2}\right)\right] \frac{1}{0.5} d v_{1} \frac{1}{0.5} d v_{2} .
\end{aligned}
$$

This expression differs from the one under full disclosure in the extra two terms at the end. We refer to these terms as information premia or side-payments, to capture that they come from the fact that now that there is possibly some discrepancy between the seller's and the buyers' beliefs, the seller can use the fact that she has superior information about a buyer's opponent to extract higher payments.

In the general analysis below, we argue that for all feasible mechanisms, given some disclosure policy $c$, it must be the case that the ex-ante expected sum of the information premia must be zero, that is

$$
\begin{aligned}
& \quad 0.25 \int_{0}^{0.5} \int_{0}^{0.5} c(m \mid L, L)\left[\tau_{1}\left(v, L, L, m_{1}, m_{2}\right)+\tau_{2}\left(v, L, L, m_{1}, m_{2}\right)\right] \frac{1}{0.5} d v_{1} \frac{1}{0.5} d v_{2} \\
& +0.25 \int_{0.5}^{1} \int_{0.5}^{1} c(m \mid H, H)\left[\tau_{1}\left(v, H, H, m_{1}, m_{2}\right)+\tau_{2}\left(v, H, H, m_{1}, m_{2}\right)\right] \frac{1}{0.5} d v_{1} \frac{1}{0.5} d v_{2} \\
& +0.25 \int_{0.5}^{1} \int_{0}^{0.5} c(m \mid L, H)\left[\tau_{1}\left(v, L, H, m_{1}, m_{2}\right)+\tau_{2}\left(v, L, H, m_{1}, m_{2}\right)\right] \frac{1}{0.5} d v_{1} \frac{1}{0.5} d v_{2} \\
& +0.25 \int_{0}^{0.5} \int_{0.5}^{1} c(m \mid H, L)\left[\tau_{1}\left(v, H, L, m_{1}, m_{2}\right)+\tau_{2}\left(v, H, L, m_{1}, m_{2}\right)\right] \frac{1}{0.5} d v_{1} \frac{1}{0.5} d v_{2} \\
& =0 .
\end{aligned}
$$

This result, as we explain in detail later, is a consequence of the incentive-compatibility constraints for the buyers, together with the fact that in our model there is a common prior and both the seller and the buyers use Bayes' rule to update their information. Now recall that we have argued that the optimal mechanism under full disclosure is feasible for all disclosure policies. This implies that the informed seller can always achieve her payoff under full disclosure. The fact that for all feasible mechanisms the ex-ante expected sum of the information premia must be zero, together with the fact that the informed seller can always achieve her payoff under full disclosure, implies $\tau_{1}(v, s, m)+\tau_{2}(v, s, m)=0$ for all $s$ and $m$ for all feasible mechanisms, given some disclosure policy $c$. Hence, we can safely ignore the extra terms of the information premium.

Given that we can now ignore the terms on information premium, the seller's revenue when she has observed $L, L$ is given by

$$
\begin{aligned}
& \int_{0}^{0.5} \int_{0}^{0.5}\left[p_{1}\left(v_{1}, v_{2}, L, L, m_{1}, m_{2}\right)\left(2 v_{1}-0.5\right)+p_{2}\left(v_{1}, v_{2}, L, L, m_{1}, m_{2}\right)\left(2 v_{2}-0.5\right)\right] \frac{1}{0.5} d v_{1} \frac{1}{0.5} d v_{2} \\
& -\underline{U}_{1}\left(0, L, L, m_{1}, m_{2}\right)-\underline{U}_{2}\left(0, L, L, m_{1}, m_{2}\right),
\end{aligned}
$$


which, apart from the fact that we now have some arbitrary messages $m_{1}, m_{2}$ entering $p_{1}$ and $p_{2}$ instead of $L, L$, is the same expression as the seller's revenue under full disclosure. ${ }^{26}$ The same is true, of course, when the seller has observed $H, H ; H, L$ and $L, H$. Hence, the schedule that we have derived as a solution when the seller employs the information disclosure policy, is a solution for every disclosure policy.

This example illustrates the main results (and the forces behind them) that lead to the information irrelevance theorem that we are about to prove. The first result is that the optimal mechanism under full disclosure is feasible for the informed seller's problem; in fact, it is feasible for all disclosure policies. The second result is that information premia for each type of the seller must be indentically equal to zero. These two results imply that the informed seller will do best by using a mechanism that maximizes the allocation-dependent part of her revenue. The solution of this problem coincides with the optimal mechanism under full disclosure irrespective of the disclosure policy employed by the seller at date 1 .

\section{Information Disclosure \& Other Mechanisms}

It is interesting to compare the effect of information disclosure when the seller employs optimal mechanisms, with cases where the seller employs specific auctions, such as a second-price or a first-price auction. First, note that the effect of information disclosure on any kind of auction procedure that has a dominant strategy equilibrium (such as a second-price auction $(S P A)$ ) is nil. (The same is true for the effect of information disclosure on the "collusive" equilibrium of the $S P A$ where all buyers, but one, bid zero - this is still an equilibrium regardless of what buyers know about their competitors.) Then, one needs to consider other auction procedures that have Bayes-Nash equilibria that are affected by the seller's information disclosure, such as a first-price auction $(F P A)$. Now we compare the effect of two extreme disclosure policies, full disclosure and no disclosure, on the equilibria of first-price auctions and show that, there, information disclosure matters.

Equilibrium of First-Price Auction under No Disclosure: When the seller discloses no information to the buyers before they bid in an FPA, at the point where they choose their bidding functions, it is common knowledge that each buyer views his competitor as having a valuation drawn from the uniform distribution on $[0,1]$. In this case, there is a symmetric equilibrium where both buyers bid according to:

$$
b(v)=\frac{v}{2} \text { for } v \in[0,0.5] .
$$

In this equilibrium, the bidder with the highest realized valuation wins the object and pays a price that is equal to half his value.

Equilibrium of First-Price Auction under Full Disclosure: Here, depending on what the seller has observed (which determines what she discloses), there are four scenarios to consider.

\footnotetext{
${ }^{26}$ They also enter the expressions of $\underline{U}_{i}$ because these terms depend on the $p_{i}^{\prime} s$, but this is immaterial because once the $p_{i}^{\prime} s$ are chosen optimally, these terms turn out to be zero.
} 
1. Seller observed $L, L:$ In this case, under full disclosure it is common knowledge that each buyer views his competitor as having a valuation drawn from the uniform distribution on $[0,0.5]$. In this case, too, there is a symmetric equilibrium where both buyers use the following bid function:

$$
b(v)=\frac{v}{2} \text { for } v \in[0,0.5] .
$$

In this equilibrium, the bidder with the highest realized valuation wins the object and pays a price that is equal to half his value.

2. Seller observed $H, H$ : In this case, under full disclosure it is common knowledge that each buyer views his competitor as having a valuation drawn from the uniform distribution on $[0.5,1]$. There is a symmetric equilibrium where both buyers use the following bid function:

$$
b(v)=\frac{v}{2}+\frac{0.25}{2 v} \text { for } v \in[0,0.5] .^{27}
$$

In this equilibrium, the bidder with the highest realized valuation wins the object (bidding strategies are strictly increasing on $(0.5,1])$.

3. Seller observed $L, H$ (analogously $H, L)$ : In this case, it is common knowledge that buyer 1 knows that buyer 2 believes that $v_{1}$ is uniformly distributed on [0,0.5]. It is also common knowledge that buyer 2 knows that buyer 1 believes that $v_{2}$ is uniformly distributed on $[0.5,1]$. Here, we have to calculate the equilibrium of an asymmetric first-price auction, where buyer 1's valuation is drawn from the uniform distribution on $[0,0.5]$ and buyer 2 's valuation is drawn from the uniform distribution on $[0.5,1]$. Using the results of Kaplan and Zamir $(2007)^{28}$, we obtain the following inverse bid functions:

$$
\begin{aligned}
& v_{1}(b)=\frac{0.25}{2.0-2.529(0.5-2 b) e^{\frac{0.5}{0.5-2 b}}-4 b} \\
& v_{2}(b)=0.5+\frac{0.25}{-4 b-1.5816(0.5-2 b) e^{-\frac{0.5}{0.5-2 b}}} .
\end{aligned}
$$

\footnotetext{
${ }^{27}$ The general formula for the bidding functions is given by $b(v)=v-\int_{\underline{v}}^{v} F^{n-1}(w) d w / F^{n-1}(v)$ for all $v \in(\underline{v}, \bar{v}]$ and $b(\underline{v})$ has support $\{$ no bid, $\underline{v}\}$. See Lebrun (1999), Corollary 3.

${ }^{28}$ The general formulas of the inverse bidding functions for environments that include this example (two bidders, uniform distributions with different supports) are described in Proposition 1 in Kaplan and Zamir (2007).
} 
The bid functions are depicted in Figure 1:

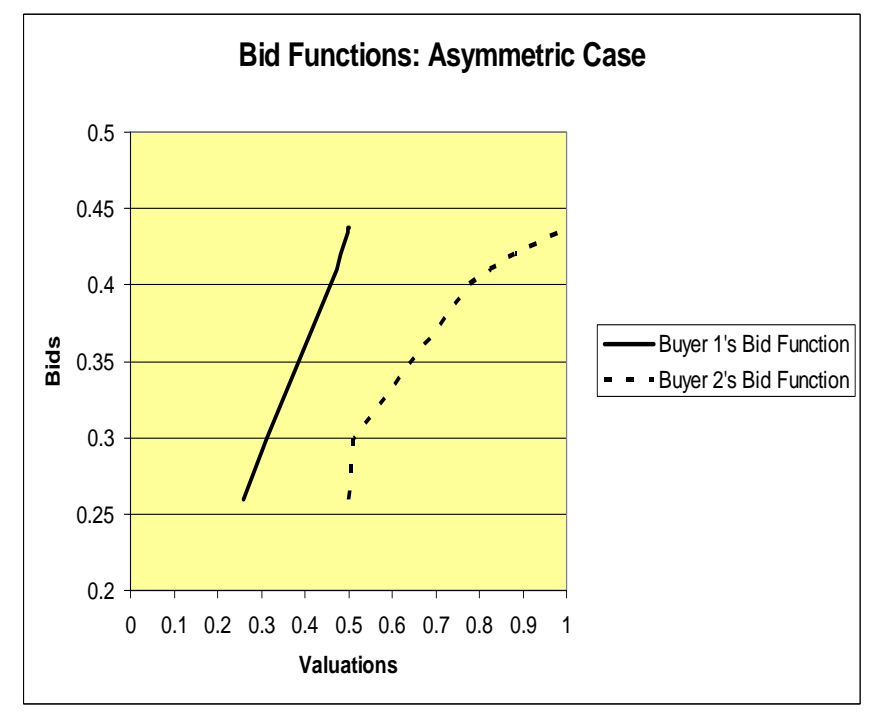

Figure 1

A quick look at the bid functions reveals that, in this case, the equilibrium is inefficient. ${ }^{29}$

From this example, one can see that whether the seller discloses none or all of her information has a dramatic effect on the equilibrium bid functions and allocations of a first-price auction. In the case of no disclosure, the equilibrium is efficient because the highest bidder wins. In the case of full disclosure, the equilibrium depends on the signals that the seller has observed. When she observes the same signal for both buyers, equilibria are efficient, but when she observes different signals they are not. In contrast, as we discussed earlier, information disclosure affects neither the outcome nor the buyers' and the seller's payoff of an optimal mechanism.

\subsection{General Analysis}

In this subsection, we establish the information irrelevance theorem. Before we do so, we outline the main steps we follow. We start by deriving necessary conditions for menus $p, x$ that satisfy the constraints for the buyers. Then, for a given disclosure policy $c$, we consider a program that ignores the seller's constraints and where each type of the seller chooses the mechanism that maximizes the allocation-dependent part of revenue, ignoring the information premium. We call this problem Program $S$. We proceed to argue that a solution to Program $S p^{*}, x^{*}$ is feasible for the mechanism selection stage for the given disclosure policy. Then, using this finding, as well as implications of the feasibility constraints for the buyers, we establish that, at a solution of the informed seller problem, the information premium must be equal to zero for each type of the seller. Hence, even

\footnotetext{
${ }^{29}$ Note that buyer 1 does not submit a bid if his valuation is below 0.2604 , which is roughly equal to the smallest bid submitted by buyer 2 (buyer 2 bids 0.26 when his valuation is 0.5 ).
} 
though the schedule $p^{*}, x^{*}$ is derived so as to maximize the allocation-dependent part of revenue, it turns out that it maximizes all terms (since the information premium term must be zero). ${ }^{30}$ We then move on to show that each solution to Program $S$ is independent of the disclosure policy employed at date 1, and it coincides with a mechanism that maximizes the seller's revenue when all her information is commonly known. From the last observation, we conclude that no matter what disclosure policy is employed at date 1, at every optimal mechanism given this disclosure policy, the seller's expected revenue at each $s$ is equal to the expected revenue of a revenue-maximizing mechanism when $s$ is common knowledge. ${ }^{31}$

\subsubsection{Implications of Feasibility}

We first investigate consequences of incentive compatibility for buyer $i, i \in I$. By the Envelope Theorem $^{32}$ we have that

$$
\frac{\partial U_{i}\left(v_{i}, s_{i}, m_{i}\right)}{\partial v_{i}}=P_{i}\left(v_{i}, s_{i}, m_{i}\right)
$$

where $P_{i}$ is bounded, so it is integrable, that is ${ }^{33}$

$$
U_{i}\left(v_{i}, s_{i}, m_{i}\right)=\int_{\underline{v}_{i}\left(s_{i}\right)}^{v_{i}} P_{i}\left(t_{i}, s_{i}, m_{i}\right) d t_{i}+U_{i}\left(\underline{v}_{i}\left(s_{i}\right), s_{i}, m_{i}\right), \text { for } v_{i} \in V_{i}\left(s_{i}\right) .
$$

Using standard arguments (see, for instance, Myerson (1981)), we can obtain the following necessary conditions for feasibility.

Lemma 1 If a mechanism $(p, x)$ satisfies $I C_{i}, P C_{i}$ and $R E S$ for all buyers $i \in I$, then the following must be true. For all $i \in I$

$$
\begin{aligned}
& U_{i}\left(\underline{v}_{i}\left(s_{i}\right), s_{i}, m_{i}\right) \geq 0, \text { for all } s_{i}, m_{i} \in S_{i} \times \mathcal{M}_{i}\left(S_{i}\right) \\
& P_{i}\left(v_{i}, s_{i}, m_{i}\right) \text { is increasing in } v_{i}, \text { for all } s_{i}, m_{i} \in S_{i} \times \mathcal{M}_{i}\left(S_{i}\right) \\
& U_{i}\left(v_{i}, s_{i}, m_{i}\right)=\int_{\underline{v}_{i}}^{v_{i}}\left(s_{i}\right) \\
& P_{i}\left(t_{i}, s_{i}, m_{i}\right) d t_{i}+U_{i}\left(\underline{v}_{i}\left(s_{i}\right), s_{i}, m_{i}\right), \text { for all } v_{i}, s_{i}, m_{i} \in V_{i}\left(S_{i}\right) \times S_{i} \times \mathcal{M}_{i}\left(S_{i}\right) \\
& p_{i}(v, s, m) \geq 0 \text { and } \Sigma_{i \in I} p_{i}(v, s, m) \leq 1, \text { for all } v, s, m \in V(S) \times S \times \mathcal{M}(S) .
\end{aligned}
$$

We now employ Lemma 1 in order to obtain a useful rewriting of the seller's expected revenue. In order to do so, we need a few additional pieces of notation. Let $J_{i}\left(v_{i}, s_{i}\right)$ denote the virtual

\footnotetext{
${ }^{30}$ In other words, instead of deriving implications from the incentive compatibility constraints for the seller, we derive a schedule that maximizes revenue for each type of the seller, and show that, is, indeed, incentive-compatible for the seller. This is analogous to the approach in Maskin and Tirole (1990), who characterize SUPO allocations, (Strong Unconstrained Pareto Optimal allocations). "Unconstrained" refers to the fact that the principal's constraints are not taken into account.

${ }^{31}$ Note that we are analyzing continuation equilibria at date 2 for a given disclosure policy $c$, which may or may not be equilibrium-feasible, we just assume that $c$ describes what the seller did at date 1 . We establish that maximal revenue generated at $t=2$ is independent of $c$ (even the ones that are not equilibrium-feasible).

${ }^{32}$ For general Envelope Theorems applicable here, see Milgrom and Segal (2002).

${ }^{33}$ Recall that valuation $\underline{v}_{i}\left(s_{i}\right)$ is the lowest possible valuation for $i$ given $s_{i}$.
} 
surplus for buyer $i$ conditional on $s_{i}$. It is given by

$$
J_{i}\left(v_{i}, s_{i}\right)=v_{i}-\frac{1-F_{i}\left(v_{i} \mid s_{i}\right)}{f_{i}\left(v_{i} \mid s_{i}\right)} .
$$

Let also $\tau_{i}(v, s, m)$ denote the information premium paid by buyer $i$, when the vector of reports is $v, s, m$. It is defined by

$$
-\tau_{i}(v, s, m) \equiv u_{i}(v, s, m)-\int_{\underline{v}_{i}\left(s_{i}\right)}^{v_{i}} p_{i}\left(t_{i}, v_{-i}, s, m\right) d t_{i}-u_{i}\left(\underline{v}_{i}\left(s_{i}\right), v_{-i}, s, m\right) .
$$

Finally, let

$$
\underline{U}_{i}\left(\underline{v}_{i}\left(s_{i}\right), s, m\right) \equiv \int_{V(s)} u_{i}\left(\underline{v}_{i}\left(s_{i}\right), v_{-i}, s, m\right) f(v \mid s) d v
$$

denote the expected payoff that accrues to $i$ from the seller's perspective, given a mechanism $p, x$, when his realized valuation is $\underline{v}_{i}\left(s_{i}\right)$, and when the seller's type is $s, m$.

The seller's revenue when her type is $s, m$ and she employs a mechanism $(p, x)$, is given by:

$$
\int_{V(s)} \Sigma_{i \in I} x_{i}(v, s, m) f(v \mid s) d v .
$$

We can rewrite revenue (7) with the help of (1) as welfare minus bidders' utilities:

$$
\int_{V(s)} \Sigma_{i \in I}\left[p_{i}(v, s, m) v_{i}-u_{i}(v, s, m)\right] f(v \mid s) d v .
$$

Then, using (5), (8) can be rewritten as

$$
\int_{V(s)} \Sigma_{i \in I}\left[p_{i}(v, s, m) v_{i}-\int_{\underline{v}_{i}\left(s_{i}\right)}^{v_{i}} p_{i}\left(t_{i}, v_{-i}, s, m\right) d t_{i}-u_{i}\left(\underline{v}_{i}\left(s_{i}\right), v_{-i}, s, m\right)+\tau_{i}(v, s, m)\right] f(v \mid s) d v .
$$

Using standard arguments (see, for example, Myerson (1981)), the above expression can be rewritten as

$$
\int_{V(s)} \Sigma_{i \in I} p_{i}(v, s, m) J_{i}\left(v_{i}, s_{i}\right) f(v \mid s) d v-\Sigma_{i \in I} \underline{U}_{i}\left(\underline{v}_{i}\left(s_{i}\right), s, m\right)+\underbrace{\int_{V(s)} \Sigma_{i \in I} \tau_{i}(v, s, m) f(v \mid s) d v}_{\text {information premium }}(9)
$$

The first two terms of the expression in (9) are standard. The new term is the last one, and it consists of the sum of extra side-payments that the seller may be able to extract for "selling" information to the buyers about their competitors. Even though the expression in (9) looks almost identical to the standard one, its derivation does not rely on the coincidence of the seller's and the buyers' beliefs, and, in addition, it does not require the seller to know anything about buyers' beliefs. Hence, the use of (5) allows us to obtain an expression of the seller's expected revenue in 
situations where the seller's and buyer $i$ 's beliefs about the valuations of $-i$ are different. ${ }^{34}$ The corresponding result in Myerson (1981) (Lemma 3) relies on the fact that the beliefs of the seller and the beliefs of $i$ about $-i$ coincide. See equation 4.10 in that paper.

We now proceed to establish a further property of incentive-compatible mechanisms for some fixed disclosure policy $c$. We show that, at a mechanism that is incentive-compatible for the buyers, the ex-ante (that is before the seller observes $s$ ) expected information premium is zero.

Proposition 1 For every information disclosure policy, and every feasible mechanism, given this disclosure policy, the ex-ante expected (from the seller's perspective) information premium incurred by buyer $i$ is zero, that is

$$
\Sigma_{s \in S} \pi(s) \int_{V(s)} \Sigma_{m \in \mathcal{M}} c(m \mid s) \tau_{i}(v, s, m) f(v \mid s) d v=0 .
$$

Proof. First, we show that for a mechanism that satisfies $I C_{i}$ for all $i \in I$, it must hold that

$$
E_{v_{-i}, s_{-i}, m_{-i}}\left[\tau_{i}(v, s, m) \mid s_{i}, m_{i}\right]=0,
$$

which can be rewritten as

$$
\Sigma_{m_{-i} \in \mathcal{M}_{-i}} \Sigma_{s_{-i} \in S_{-i}} \int_{V_{-i}\left(s_{-i}\right)} \tau_{i}(v, s, m) \frac{\pi_{i}\left(s_{i}\right) \pi_{-i}\left(s_{-i}\right) c(m \mid s)}{\pi_{i}\left(s_{i}\right) \Sigma_{s_{-i}} \pi_{-i}\left(s_{-i}\right) \Sigma_{m_{-i}} c(m \mid s)} f_{-i}\left(v_{-i} \mid s_{-i}\right) d v_{-i}=0 .
$$

To see this, note that, by definition, at a truth-telling equilibrium it must be the case that

$$
U_{i}\left(v_{i}, s_{i}, m_{i}\right)=E_{v_{-i}, s_{-i}, m_{-i}}\left[u_{i}(v, s, m) \mid s_{i}, m_{i}\right] .
$$

Now, observe that (3) can be rewritten as

$$
U_{i}\left(v_{i}, s_{i}, m_{i}\right)=E_{v_{-i}, s_{-i}, m_{-i}}\left[\int_{\underline{v}_{i}\left(s_{i}\right)}^{v_{i}} p_{i}\left(t_{i}, v_{-i}, s, m\right) d t_{i}+u_{i}\left(\underline{v}_{i}\left(s_{i}\right), v_{-i}, s, m\right) \mid s_{i}, m_{i}\right] .
$$

Combining (13) and (14) we obtain that

$$
E_{v_{-i}, s_{-i}, m_{-i}}\left[u_{i}(v, s, m)-\int_{\underline{v}_{i}\left(s_{i}\right)}^{v_{i}} p_{i}\left(t_{i}, v_{-i}, s, m\right) d t_{i}-u_{i}\left(\underline{v}_{i}\left(s_{i}\right), v_{-i}, s, m\right) \mid s_{i}, m_{i}\right]=0 .
$$

With the help of (5), (15) can be rewritten as $E_{v_{-i}, s_{-i}, m_{-i}}\left[\tau_{i}(v, s, m) \mid s_{i}, m_{i}\right]=0$ establishing (12).

Now (12) is equivalent to

$$
\Sigma_{m_{-i} \in \mathcal{M}_{-i}} \Sigma_{s_{-i} \in S_{-i}} \pi_{i}\left(s_{i}\right) \pi_{-i}\left(s_{-i}\right) \int_{V_{-i}\left(s_{-i}\right)} \tau_{i}(v, s, m) c(m \mid s) f_{-i}\left(v_{-i} \mid s_{-i}\right) d v_{-i}=0
$$

\footnotetext{
${ }^{34}$ All the arguments leading to (9) are also applicable in cases where the differences in the seller's and the buyers' beliefs result from the lack of a common prior. In this paper, even though we start with a common prior that is common knowledge, after the seller releases information, buyers may no longer know the beliefs of their competitors.
} 
for all $v_{i}, m_{i}$ and $s_{i}$. Adding over all $v_{i}, m_{i}, s_{i}$ we get (10).

As mentioned earlier, a disclosure policy induces discrepancies in beliefs. It may also induce correlations in types, even in an environment where prior information is statistically independent. From Proposition 1, we concluded that from the ex-ante perspective, irrespectively from the disclosure policy, the ex-ante expected information premium is zero. Hence, exploiting the discrepancy in beliefs, is not beneficial for the seller, at least from the ex-ante perspective.

Because the $\tau_{i}$ 's can be interpreted as a side-payment that the seller can extract from the buyers, Proposition 1 can be understood in the framework of the following coin-tossing example. There are two individuals tossing two different coins. Each individual observes the outcome of his own coin. There is a principal that observes both outcomes of the coins and discloses information as follows. Each vector of coin outcomes is mapped to a vector of private reports - one to each individual - about the realized outcome of the other person's coin. More specifically, let $H_{i}$ denote the outcome "heads" of coin $i$, and $T_{i}$ denote the outcome "tails" of coin $i$. The principal sends to each individual $i$ a message about the realization of $j$ 's coin for $i, j=1,2$; the messages are $\hat{H}_{j}, \hat{T}_{j}$. For instance, $c\left(\hat{T}_{2}, \hat{T}_{1} \mid H_{1}, H_{2}\right)$ denotes the probability that each agent receives the information that the other agent's coin turned out to be tails, $T$, when actually both coins are heads, $H$. The principal offers contingent payments, $\tau_{i}$ 's, that can depend both on the vector of the realized coin outcomes and on the vector of messages sent to the individuals. It is relatively easy to see that if contingent payments have positive expected value for the principal, they have negative expected value for at least one of the agents. Hence, the only contracts willingly accepted have, at most, zero expected value for the principal. For the same reasons as in this example, namely because there is a common prior and agents form posterior beliefs consistent with Bayes' rule, it is not possible for the seller's information premium to have strictly positive expected value.

\subsubsection{Mechanism Selection}

With the implications of the feasibility constraints in hand, we now move on to analyze the mechanism selection stage for a given disclosure policy $c$. We proceed as follows. We ignore the seller's constraints and examine mechanisms that maximize the seller's expected revenue for each of her types - that is, for each couple $s, m$ subject to the resource and the constraints for the buyers. We break this problem in two steps: First, we examine what mechanisms maximize the first two terms of (9), and then we examine what is the highest feasible possible value for the third term. The problem of maximizing the first two terms of (9) is called Program $S$, and it is given by:

subject to :

$$
\max _{p} \int_{V(s)} \Sigma_{i \in I} p_{i}(v, s, m) J_{i}\left(v_{i}, s_{i}\right) f(v \mid s) d v-\Sigma_{i \in I} \underline{U}_{i}\left(\underline{v}_{i}\left(s_{i}\right), s, m\right)
$$

$(R E S) p_{i}(v, s, m) \geq 0$ and $\Sigma_{i \in I} p_{i}(v, s, m) \leq 1$ for $v, s, m \in V(S) \times S \times \mathcal{M}(S)$

(IC) $P_{i}\left(v_{i}, s_{i}, m_{i}\right)$ increasing in $v_{i}$, for all $s_{i}, m_{i} \in S_{i} \times \mathcal{M}_{i}\left(S_{i}\right)$ and all $i \in I$

$(P C) \underline{U}_{i}\left(\underline{v}_{i}\left(s_{i}\right), s, m\right) \geq 0$ for all $s, m \in S \times \mathcal{M}(S)$ and all $i \in I$ 
The first set of constraints of Program $S$ are resource constraints; the second and third sets are necessary conditions for incentive compatibility and the participation constraints for the buyers. A solution of Program $S$ gives an allocation rule $p^{*}$ from which we obtain the payment rule using $x_{i}^{*}(v, s, m)=p_{i}^{*}(v, s, m) v_{i}-\int_{\underline{v}_{i}\left(s_{i}\right)}^{v_{i}} p_{i}^{*}\left(t_{i}, v_{-i}, s, m\right) d t_{i}$. In the steps below, when we refer to a solution of Program $S$, we ignore the term $-\Sigma_{i \in I} \underline{U}_{i}\left(\underline{v}_{i}\left(s_{i}\right), s, m\right)$ since it turns out to be zero at an optimum.

We first establish that a solution of Program $S$ is a feasible choice for the seller, given $c$.

Lemma 2 A solution $p^{*}, x^{*}$ of Program $S$, given some disclosure policy c, is feasible for the seller's mechanism selection game at date 2.

Proof. Given that $p^{*}, x^{*}$ is a solution of Program $S$ for each $s, m$, it satisfies resource constraints, as well as all the constraints for the buyers. Now, it is easy to see that it satisfies the participation constraints of the seller, since the seller can always guarantee zero by not assigning the objects and not charging anything. Hence, the value of Program $S$ is positive for each $s, m$. Then, we just need to establish incentive compatibility for the seller. This is also immediate, since $p, x$ solves Program $S$ for each $s, m$, which implies that, by misreporting, the seller can select only a schedule that maximizes a "wrong "objective function.

We now use Proposition 1 and Lemma 2 to establish that, at a solution of our informed seller problem, it must hold that $\int_{V(s)} \tau_{i}(v, s, m) f(v \mid s) d v=0$ for all $i \in I$ and all $s, m \in S \times \mathcal{M}(S)$.

Proposition 2 Given any disclosure policy, at a solution of our informed seller problem, it must hold that $\Sigma_{i \in I} \int_{V(s)} \tau_{i}(v, s, m) f(v \mid s) d v=0$ for all $i \in I$ and all $s, m \in S \times \mathcal{M}(S)$.

Proof. Let $p, x$ denote a solution for our informed seller, given some disclosure policy $c$. From (10) it follows that if for some $s, m \in S \times \mathcal{M}(S)$ we have that $\Sigma_{i \in I} \int_{V(s)} \tau_{i}(v, s, m) f(v \mid s) d v>0$, then, there exists another $\hat{s}, \hat{m} \in S \times \mathcal{M}(S)$ with

$$
\Sigma_{i \in I} \int_{V(s)} \tau_{i}(v, \hat{s}, \hat{m}) f(v \mid s) d v<0 .
$$

From Lemma 2, we know that $p^{*}, x^{*}$ is feasible for the informed seller, given $c$. Then, if $p, x$ is a solution to the informed seller problem for $c$, then it must be the case that for each $s, m$, the seller's revenue at $p, x$ is as least as high as at $p^{*}, x^{*}$ for all $s, m$. For type $\hat{s}, \hat{m}$, this implies that

$$
\begin{aligned}
& \int_{V(s)} \Sigma_{i \in I} p_{i}(v, \hat{s}, \hat{m}) J_{i}\left(v_{i}, s_{i}\right) f(v \mid s) d v-\Sigma_{i \in I} \underline{U}_{i}(\hat{s}, \hat{m})+\int_{V(s)} \Sigma_{i \in I} \tau_{i}(v, \hat{s}, \hat{m}) f(v \mid s) d v \\
\geq & \int_{V(s)} \Sigma_{i \in I} p_{i}^{*}(v, \hat{s}, \hat{m}) J_{i}\left(v_{i}, s_{i}\right) f(v \mid s) d v .
\end{aligned}
$$

(Each type of seller has the option of using $p^{*}, x^{*}$, irrespective of the disclosure policy.) But because $p^{*}$ solves Program $S$, it satisfies

$\int_{V(s)} \Sigma_{i \in I} p_{i}^{*}(v, \hat{s}, \hat{m}) J_{i}\left(v_{i}, s_{i}\right) f(v \mid s) d v \geq \int_{V(s)} \Sigma_{i \in I} p_{i}(v, \hat{s}, \hat{m}) J_{i}\left(v_{i}, s_{i}\right) f(v \mid s) d v-\Sigma_{i \in I} \underline{U}_{i}\left(\underline{v}_{i}\left(s_{i}\right), \hat{s}, \hat{m}\right)$, 
which together with (16) contradicts (17).

This Proposition tells us that the best that the seller can hope for is a mechanism where for each $s, m$ it solves Program $S$.

A solution of Program $S$ may depend on the disclosure policy, because it affects buyers' beliefs, which, in turn, determine the set of incentive-compatible assignment rules. We now establish that, irrespective of the disclosure policy, a solution of Program $S, p^{*}, x^{*}$ coincides with a mechanism chosen by the seller when $s$ is commonly known; call it $p^{s}, x^{s}$, that is

$$
\begin{aligned}
& p^{*}(v, s, m)=p^{s}(v) \\
& x^{*}(v, s, m)=x^{s}(v) .
\end{aligned}
$$

Remark 1 In what follows, we are assuming that for all $i \in I, J_{i}\left(v_{i}, s_{i}\right)=v_{i}-\frac{1-F_{i}\left(v_{i} \mid s_{i}\right)}{f_{i}\left(v_{i} \mid s_{i}\right)}$ is strictly increasing in $v_{i}$. If not, we can replace each $J_{i}$ with an appropriately constructed increasing function $\hat{J}_{i}$ (this procedure is sometimes referred to as "ironing"35) and proceed exactly along the lines described in Myerson (1981). ${ }^{36}$

Proposition 3 For each $s$, a solution of Program $S$ is independent from the disclosure policy, and it coincides with a solution of the seller's problem when $s$ is common knowledge. Moreover, it is feasible for the seller's mechanism selection game at date 2, no matter the disclosure policy employed at date 1 .

Proof. The expression $\Sigma_{i \in I} p_{i}(v, s, m) J_{i}\left(v_{i}, s_{i}\right)$ is maximized pointwise - that is, for each vector $(v, s, m)$ - by setting

$$
\begin{aligned}
& p_{i}^{*}(v, s, m)=1 \text { if } J_{i}\left(v_{i}, s_{i}\right) \geq J_{j}\left(v_{j}, s_{j}\right), \text { all } j \neq i \text { and } J_{i}\left(v_{i}, s_{i}\right) \geq 0 \\
& p_{i}^{*}(v, s, m)=0, \text { otherwise. }
\end{aligned}
$$

In the case of ties, the object is awarded to the lowest index buyer among the ones who tie.

From (20) it also immediately follows that $p^{*}$ coincides with the assignment rule that the seller would choose when $s$ is common knowledge; hence, (19) holds.

We now proceed to establish that $p^{*}, x^{*}$ is feasible for the seller and for the buyers for every disclosure policy.

First, we argue that $p^{*}, x^{*}$ satisfies the buyers' constraints irrespective of the disclosure policy. This is because, $p_{i}^{*}(v, s, m)$ is increasing in $v_{i}$ for all $v_{-i}, s, m$, implying that $p^{*}, x^{*}$ is dominant strategy incentive-compatible. Moreover, since $u_{i}\left(\underline{v}_{i}\left(s_{i}\right), v_{-i}, s, m\right)=0$ for all $v_{-i}, s, m$ and all $i \in I$ it satisfies the participation constraints irrespective of the buyers' beliefs, which are what the

\footnotetext{
${ }^{35}$ The ironed virtual valuation is obtained in essentially three steps: First, we integrate the virtual valuation; second we convexify this integral by finding the "highest" convex function that lies (weakly) below the integral; and, finally, we differentiate the convexified integral. The function obtained in this way is increasing because it is the derivative of a convex function and often referred to as the "ironed" virtual valuation.

${ }^{36}$ As is shown, in Skreta (2007), we can iron virtual surpluses also in environments where $f_{i}$ fails to be continuous and strictly positive.
} 
disclosure policy affects. Now, to see that $p^{*}, x^{*}$ is feasible for the seller irrespective of the disclosure policy, notice that the objective function of Program $S$ does not depend on the disclosure policy employed at date 1. Also, from (19) one can see that $p^{*}, x^{*}$, is independent from $m$, and hence from the disclosure policy. Therefore, $p^{*}, x^{*}$ is feasible for the seller's mechanism selection game at date 2 , no matter the disclosure policy employed at date 1 .

With this result in hand, we are now ready to state and prove our information irrelevance theorem:

Theorem 1 Fix a disclosure policy. At every optimal mechanism given this disclosure policy, the seller's expected revenue at each $s$ is equal to the expected revenue of a revenue-maximizing mechanism when $s$ is common knowledge.

Proof. This theorem follows from Propositions 2 and 3. In Proposition 2, we concluded that the best that the seller can hope for is a mechanism where for each $s, m$, it solves Program $S$. Proposition 3 then established that a solution of Program $S$ is independent from the disclosure policy, and it coincides with a solution of the seller's problem when $s$ is common knowledge. It also established that it is feasible for the seller's mechanism selection game at date 2, no matter the disclosure policy employed at date 1.

Now, let us offer a few connections between our finding and the earlier literature on informed principal. Because $p^{*}, x^{*}$ satisfies the incentive constraints for the seller when the buyers know the seller's type (since we showed that it is feasible, irrespective of the disclosure policy), it is safe in the terminology of Myerson (1983). By construction, it is also interim-efficient $\left(S U P O^{37}\right.$ in the terminology of Maskin and Tirole (1990)), thus, it is a strong solution, in the sense of Myerson (1983). As argued in the works of Maskin and Tirole (1990 and 1992), strong solutions are equilibria of the three-stage mechanism selection game, and they survive all common refining criteria.

\subsubsection{Discussion}

We have established an information irrelevance result. For every disclosure policy, a revenuemaximizing mechanism chosen by the informed seller must generate at each $s, m$ exactly the same revenue that is generated by the mechanism that is optimal when $s$ is commonly known. This is despite the fact that the seller can use her private information to create correlations in the buyers' types.

We now highlight the forces behind our result. We discuss which of the them are present in more general mechanism design problems where, for instance, values can be interdependent or common, and, which ones are more specific to this standard independent private value scenario.

\footnotetext{
${ }^{37}$ An allocation is $S U P O$, from the point of view of different types of the principal for given beliefs (of the agent), if there exists no other feasible allocation, (satisfying the constraints for the agent), even for different beliefs, that Pareto dominates it, (Maskin and Tirole (1990)).
} 
The key forces of the result are three. First, disclosure policies, irrespective of how sophisticated they are, do not eliminate information rents. Second, the seller's incentive constraints are not binding. And, third, when looking at the relaxed program for the seller, namely Program $S$, its solution is dominant-strategy incentive-compatible. As we discuss below, the first two forces are present in more general mechanism design problems; however, the last one is more particular to this simple independent private value problem.

Disclosure Policies Create Correlation in Types, but do not Eliminate Information Rents: From our analysis, it follows that information-disclosure policies do not affect the information rents that each type of buyer enjoys and that are always equal to the ones obtained when the seller's information is fully disclosed. Buyer $i$ 's expected payoff, given a disclosure policy $c$ and a mechanism that is incentive compatible given $c$, is given (3). From (3) we have that $U_{i}\left(v_{i}, s_{i}, m_{i}\right) \geq 0$, for all $v_{i} \in V_{i}\left(s_{i}\right)$. Moreover, if $v_{i}^{\min }\left(s_{i}\right)$ is the lowest type for which $P_{i}\left(t_{i}, s_{i}, m_{i}\right)>0$, then for $v_{i} \in\left[v_{i}^{\min }\left(s_{i}\right), \bar{v}_{i}\left(s_{i}\right)\right]$ we have that $U_{i}\left(v_{i}, s_{i}, m_{i}\right)>0$. Therefore, irrespective of the sophistication of the seller's information-disclosure policies, for types that receive the object with strictly positive probability, full surplus extraction from buyer $i$ 's perspective is not possible, unless he has no private information, which can happen when $s_{i}$ fully reveals $i$ 's valuation to the seller. This observation may sound a bit surprising since, for most information-disclosure policies, buyers' types become correlated. However, full surplus extraction is impossible because more than one payoff-relevant types have the same beliefs. Here, the set of payoff-relevant types, that is the $v_{i}$ 's, that are associated with the same probability distribution over belief-relevant parts, namely $s_{i}, m_{i}$, belong in the set $V_{i}\left(s_{i}\right)=\left[\underline{v}_{i}\left(s_{i}\right), \bar{v}_{i}\left(s_{i}\right)\right]$. Then, irrespective of the disclosure policy, all $v_{i}$ 's in $V_{i}\left(s_{i}\right)$ can mimic each other exactly as in the case where $s_{i}$ was commonly known. Hence,

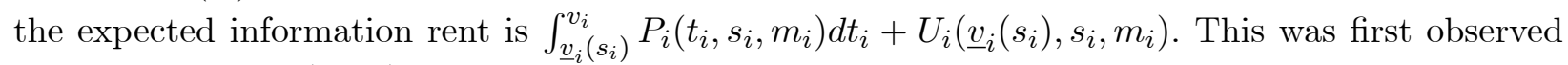
by Zvika Neeman (2004).

However, this observation, on its own, does not immediately imply that disclosure policies do not affect information rents. The reason is that expected information rent in (3) is calculated with $i$ 's beliefs and these beliefs may differ from the seller's. What is relevant for the seller's is "how often" $u_{i}(v, s, m)>\int_{\underline{v}_{i}\left(s_{i}\right)}^{v_{i}} p_{i}\left(t_{i}, v_{-i}, s, m\right) d t_{i}+u_{i}\left(\underline{v}_{i}\left(s_{i}\right), v_{-i}, s, m\right)$ versus the reverse, from her perspective. In the first part of the proof of Proposition 1, we established that, from a buyer's perspective, the expected difference, (slack), of $u_{i}(v, s, m)$ and $\int_{\underline{v}_{i}\left(s_{i}\right)}^{v_{i}} p_{i}\left(t_{i}, v_{-i}, s, m\right) d t_{i}+u_{i}\left(\underline{v}_{i}\left(s_{i}\right), v_{-i}, s, m\right)$, namely the $\tau_{i}(v, s, m)$, must be zero. We then proceeded to establish that the same is true from the seller's perspective. From these considerations, it follows that, irrespective of the disclosure policy the expected "slack" is always the same and equal to zero. This result is due to the fact that we are in a consistent model with a common prior, and buyers form beliefs according to Bayes' rule. This feature will be also present in all models where there is a common prior and initial information is statistically independent, irrespective of whether values are private or interdependent.

The Seller's Constraints are Not Binding: From our analysis, it became clear that the seller's incentive constraints were not binding. This feature is due to the fact that the information 
that the seller has is semi-private, in the sense that it is not exclusive, (remember that buyer $i$ knows the signal that the seller has about himself). This force is present in more general mechanism design problems, such as the ones that we consider in the next section.

Disclosure Policies do not Affect the Value of Program $S$ : The reason behind this result is that in this baseline $I P V$ model, a solution of Program $S$ is dominant-strategy incentivecompatible. ${ }^{38}$ However, it is not true in more general mechanism design problems. This last reason is what makes all information-disclosure policies equivalent in the environment we have examined so far. As we show in the next section, in more general mechanism design problems, information disclosure policies may matter, because they affect the set of incentive-compatible mechanisms, which, in turn, affect the value of Program $S$. For those cases we show that the value of Program $S$ is maximized when the seller discloses no information at all.

\section{General Environment: Interdependent Values, Non-linear Payoffs, Multiple} Goods.

Here, we examine revenue-maximizing mechanisms in more general environments that allow for interdependent or common values and for multiple objects. There is a risk-neutral seller who faces $I$ risk-neutral buyers. Let $Z$ denote the set of allocations - that is, the set of all possible assignments of the $\operatorname{good}(\mathrm{s}) .{ }^{39}$ Both $Z$ and $I$ are finite natural numbers. Buyer $i$ 's payoff from allocation $z$ is denoted by $u_{i}^{z}\left(v_{i}, v_{-i}\right)$, and it depends on $v_{i}$, and on the valuations of all the other buyers $v_{-i}$. This formulation allows for values to be private, interdependent, or purely common. We assume that, for all $i \in I, u_{i}^{z}\left(\cdot, v_{-i}\right)$ is increasing, convex and differentiable for each $z$ and $v_{-i} \cdot{ }^{40}$ A buyer's payoff from not participating in the mechanism is taken to be zero. We also normalize the seller's payoff from all $z \in Z$ to be zero.

As in the baseline model, the $v_{i}$ 's are statistically independent from one another. Also, the structure of the signals that the seller observes and the definition of the disclosure policy are all as in the baseline model. For the same reasons as there, we appeal to the revelation and the inscrutability principles.

We need to adjust the definition of direct revelation mechanisms only slightly. Here, the assignment rule $p: V(S) \times S \times \mathcal{M}(S) \longrightarrow \Delta(Z)$ specifies the probability of each allocation for a given vector of reports. We denote by $p_{z}(v, s, m)$ the probability that allocation $z$ is implemented when

\footnotetext{
${ }^{38}$ This feature is not unique to this problem. Mookherjee and Reichelstein (1992) describe environments where an optimal Bayesian incentive-compatible mechanism is dominant strategy incentive-compatible.

${ }^{39}$ In the case that the seller has $1 \leq N<\infty$ objects for sale, an allocation $z$ is an assignment of the objects to the buyers and to the seller. It is a vector with $N$ components, where each component stands for an object and it specifies who gets it; therefore, the set of possible allocations is finite, and given by $Z \subseteq[I \cup\{0\}]^{N}$. Note that the formulation is very flexible and allows for the goods to be heterogeneous, substitutes for some buyers, whereas complements for other buyers and for externalities.

${ }^{40}$ This model shares common features to the one considered by Figueroa and Skreta (2007).
} 
the vector of reports is $v, s, m$.

With some abuse of notation, we now let $u_{i}(v, s, m)=\Sigma_{z \in Z} p_{z}(v, s, m) u_{i}^{z}\left(v_{i}, v_{-i}\right)-x_{i}(v, s, m)$, and $U_{i}\left(v_{i}, s_{i}, m_{i}\right)=\max _{v_{i}^{\prime}} E_{v_{-i}, s_{-i}, m_{-i}}\left[\Sigma_{z \in Z} p_{z}\left(v_{i}^{\prime}, v_{-i}, s, m\right) u_{i}^{z}\left(v_{i}, v_{-i}\right)-x_{i}\left(v_{i}^{\prime}, v_{-i}, s, m\right) \mid s_{i}, m_{i}\right]$, stand for their analogs in this more general environment. Notice that $U_{i}\left(v_{i}, s_{i}, m_{i}\right)$ is convex, since it is a maximum of convex functions.

The statements of the constraints for the seller and the buyers are analogous to the standard $I P V$ model. Then, by using similar arguments as there, one can establish a result parallel to Lemma 1. In the present context, the incentive constraints translate into the requirement that the derivative of $U_{i}$, namely,

$$
P_{i}\left(v_{i}, s_{i}, m_{i}\right) \equiv E_{v_{-i}, s_{-i}, m_{-i}}\left[\Sigma_{z \in Z} p_{z}(v, s, m) \frac{\partial u_{i}^{z}\left(v_{i}, v_{-i}\right)}{\partial v_{i}} \mid s_{i}, m_{i}\right],
$$

evaluated at the true type is weakly increasing. ${ }^{41}$

Similarly, as in the baseline model, we define virtual surpluses. This concept is now allocationspecific, because an allocation may be affecting all buyers, and it is given by

$$
J_{z}(v, s) \equiv \Sigma_{i \in I}\left[u_{i}^{z}\left(v_{i}, v_{-i}\right)-\frac{1-F_{i}\left(v_{i} \mid s_{i}\right)}{f_{i}\left(v_{i} \mid s_{i}\right)} \frac{\partial u_{i}^{z}\left(v_{i}, v_{-i}\right)}{\partial v_{i}}\right] .
$$

The seller's revenue, when her type is $s, m$, is given by $\int_{V(s)} \Sigma_{i \in I} x_{i}(v, s, m) f(v \mid s) d v$, which by using similar arguments as in the baseline case, can be rewritten as

$$
\int_{V(s)} \Sigma_{z \in Z} p_{z}(v, s, m) J_{z}(v, s) f(v \mid s) d v-\Sigma_{i \in I} \underline{U}_{i}\left(\underline{v}_{i}\left(s_{i}\right), s, m\right)+\underbrace{\int_{V(s)} \Sigma_{i \in I} \tau_{i}(v, s, m) f(v \mid s) d v}_{\text {expected information premium }},
$$

where, here, $\tau_{i}(v, s, m)$ and $\underline{U}_{i}\left(\underline{v}_{i}\left(s_{i}\right), s, m\right)$ stand for the analogs of (5) and (6), which are given by $-\tau_{i}(v, s, m) \equiv u_{i}(v, s, m)-\int_{\underline{v}_{i}\left(s_{i}\right)}^{v_{i}} \Sigma_{z \in Z} p_{z}\left(t_{i}, v_{-i}, s, m\right) \frac{\partial u_{i}^{z}\left(t_{i}, v_{-i}\right)}{\partial v_{i}} d t_{i}-u_{i}\left(\underline{v}_{i}\left(s_{i}\right), v_{-i}, s, m\right)$ and $\underline{U}_{i}\left(\underline{v}_{i}\left(s_{i}\right), s, m\right) \equiv \int_{V(s)} u_{i}\left(\underline{v}_{i}\left(s_{i}\right), v_{-i}, s, m\right) f(v \mid s) d v$.

As in the baseline case, we analyze the mechanism-selection stage for a given disclosure policy as follows. We ignore the seller's constraints and examine mechanisms that maximize the seller's expected revenue for each of her types - that is, for each couple $s, m$ subject to the resource and the constraints for the buyers. Again, we break this problem in two steps: First, we examine which mechanisms maximize the first two terms of (22), and then we examine what is the highest feasible possible value for the third term. The problem of maximizing the first two terms of (22) is called Program $S_{G}$, and it is given by:

$$
\begin{aligned}
& \max _{p} \int_{V(s)} \Sigma_{z \in Z} p_{z}(v, s, m) J_{z}(v, s) f(v \mid s) d v-\Sigma_{i \in I} \underline{U}_{i}\left(\underline{v}_{i}\left(s_{i}\right), s, m\right) \\
& (R E S) p_{i}(v, s, m) \geq 0 \text { and } \Sigma_{i \in I} p_{i}(v, s, m) \leq 1 \text { for } v, s, m \in V(S) \times S \times \mathcal{M}(S) \\
& (I C) P_{i}\left(v_{i}, s_{i}, m_{i}\right) \text { increasing in } v_{i}, \text { for all } s_{i}, m_{i} \in S_{i} \times \mathcal{M}_{i}\left(S_{i}\right) \text { and all } i \in I \\
& (P C) \underline{U}_{i}\left(\underline{v}_{i}\left(s_{i}\right), s, m\right) \geq 0 \text { for all } s, m \in S \times \mathcal{M}(S) \text { and all } i \in I
\end{aligned}
$$

\footnotetext{
${ }^{41}$ More precisely, this holds for a selection from its subgradient, which is single-valued almost surely.
} 
Notice that Proposition 1 applies directly to this more general environment. The same is true for Lemma 2 and Proposition 2. This can be easily seen by going through their corresponding proofs and by replacing $\int_{V(s)} \Sigma_{i \in I} p_{i}(v, s, m) J_{i}\left(v_{i}, s_{i}\right) f(v \mid s) d v$ with $\int_{V(s)} \Sigma_{z \in Z} p_{z}(v, s, m) J_{z}(v, s) f(v \mid s) d v$. Hence, even in this more general problem, at a solution of the mechanism selection game at date 2 , the information premium for each type of the seller is zero, and the best that each type of the seller can achieve is a mechanism that solves Program $S_{G}$. The only difference from the standard $I P V$ case, is that solutions of Program $S_{G}$ typically depend on the disclosure policy.

If the value of Program $S_{G}$ is independent of the disclosure policy, then it is straightforward to see that our information irrelevance Theorem applies. Now, an important question is: When is the value of Program $S_{G}$ independent of the disclosure policy? A class of situations where this is true, is when its solution is dominant strategy incentive-compatible. ${ }^{42}$ This was the situation in the standard independent private-value model we examined previously. But there can be situations, where the value of Program $S_{G}$ is independent of the disclosure policy, however its solution is not dominant strategy incentive- compatible. ${ }^{43}$ In general, however, the value of Program $S_{G}$ does depend on the disclosure policies. This can happen even in a private value setup, as well as in "richer" problems with interdependent values, multiple goods, complementarities, externalities, etc. In Appendix B, we present an example where the value of Program $S_{G}$ depends on the disclosure policy in a private-value setting.

When the value of Program $S_{G}$ depends of the disclosure policy, the question that naturally arises is which disclosure policy is best. We show that the value of Program $S_{G}$ is maximized when the seller discloses no information. The reason is that any mechanism that is incentive-compatible under any alternative disclosure policy, is also incentive-compatible under the "no information disclosure" policy.

Proposition 4 All mechanisms that are feasible for Program $S_{G}$ under every disclosure policy, are also feasible for Program $S_{G}$ under the "no information" disclosure policy; hence, the value of Program $S_{G}$ is maximized under the "no information" disclosure policy.

From Proposition 4, it follows that the value of Program $S_{G}$ is maximized when the seller discloses no information. We call this disclosure policy $c^{*}$. The intuition for this result is that more

\footnotetext{
${ }^{42}$ The solution of Program $S_{G}$ is dominant strategy incentive-compatible if the following is true: If $z_{1}, z_{2} \in Z$ are any two allocations such that, for a given realization of valuations $\left(v_{i}, v_{-i}\right)$, we have $z_{1} \in \arg \max _{z \in Z} J_{z}\left(v_{i}^{-}, v_{-i}\right)$ and $z_{2} \in \arg \max _{z \in Z} J_{z}\left(v_{i}^{+}, v_{-i}\right)$ (the notation $v_{i}^{-}$means limit from the left to $v_{i}$ and $v_{i}^{+}$means limit from the right to $v_{i}$ ) then it holds that for all $v_{-i}$

$$
\frac{\partial u_{i}^{z_{2}}\left(v_{i}, v_{-i}\right)}{\partial v_{i}} \geq \frac{\partial u_{i}^{z_{1}}\left(v_{i}, v_{-i}\right)}{\partial v_{i}}
$$

These conditions are quite stringent, but they are only necessary and not sufficient conditions for Program $S_{G}$ to have a solution that is dominant strategy incentive-compatible. An easy way to see that they are not sufficient is to consider the standard independent private-value model when virtual valuations fail to be monotonic. In such cases, it is possible that the conditions we state are violated, but the optimal auction is still dominant strategy incentive-compatible.

${ }^{43}$ An example where this occurs is available from the author upon request.
} 
information makes more deviations for the buyers feasible, making, in some sense, the incentive constraints harder to satisfy. This idea is also discussed in Myerson (1986).

Suppose that the seller uses $c^{*}$, and let $p^{* *}(., s), x^{* *}(., s)$ denote a solution of Program $S_{G}$, when the seller's information is $s .{ }^{44}$ In the Theorem that follows, we establish that the mechanism $p^{* *}, x^{* *}$, where for each $s$ it is equal to $p^{* *}(., s), x^{* *}(., s)$, is a solution to our informed seller's problem.

Theorem 2 In the general case, an informed seller maximizes revenue by disclosing no information, and by employing a mechanism $p^{* *}, x^{* *}$, where for each $s$ it solves Program $S_{G}$ given this disclosure policy.

Proof. Given that the value of Program $S_{G}$ is maximal under the no information disclosure policy, $c^{*}$, we now argue that the mechanism $p^{* *}, x^{* *}$ is a solution to our informed seller problem.

At date 1, the seller discloses no information. At date 2, stage one, the seller proposes $p^{* *}, x^{* *}$ and at stage two, the buyers accept or reject $p^{* *}, x^{* *}$. And, finally, at stage three, the buyers and the seller report their types truthfully. To see that $p^{*}, x^{*}$ is an equilibrium mechanism of this game, note that given that $p^{* *}, x^{* *}$ satisfies $I C$ for both the buyers and the seller, they find it optimal to reveal their information truthfully at stage three. Also, since $p^{* *}, x^{* *}$ satisfies $P C$ for the buyers, they all find it optimal to participate at stage 2 . Now, at stage 1 , suppose that the seller proposes an out-of-equilibrium mechanism. No matter what the buyers may infer from that, the alternative schedule cannot be strictly better than $p^{* *}, x^{* *}$, no matter which types choose to deviate. This is because $p^{* *}, x^{* *}$ (given beliefs determined by the no information disclosure policy) gives the type- $s$ seller the highest expected revenue that she can hope for. Hence, no alternative $\tilde{p}, \tilde{x}$ makes the type- $s$ seller strictly better-off than $p^{* *}, x^{* *}$. Therefore, $p^{* *}, x^{* *}$ is an equilibrium allocation.

In terms of comparison with the earlier literature on informed principal, note that the allocation induced by the mechanism $p^{* *}, x^{* *}$ is $S U P O$ in the terminology of Maskin and Tirole (1990). It is $U P O$ for the beliefs equal to the prior, ${ }^{45}$ and there is no other $U P O$, even for other posterior beliefs, that is better than $p^{* *}, x^{* *}$. Note, however, that $p^{* *}, x^{* *}$ need not be the only solution of Program $S_{G}$, given the no information disclosure policy. When there is more than one solution, all of them are $S U P O$. We close in the next section with a few concluding remarks.

\section{Concluding Remarks}

This paper studies revenue-maximizing auctions by an informed seller. The seller has information about various buyers that is not known to all buyers participating in the auction. Before the auction, the seller can disclose any amount of information she sees fit. After disclosing information, the seller chooses a revenue-maximizing mechanism. At that point, we have an informed seller problem.

\footnotetext{
${ }^{44}$ We remove the argument of $m$ from $p^{* *}, x^{* *}$ because the vector $v, s$ summarizes all relevant information, when the seller discloses no information.

${ }^{45}$ These are the beliefs of the buyers at phase two, after the seller discloses no information at phase one.
} 
For the classical single-object independent private-values environment, we establish an informationirrelevance theorem. The release of information correlated with buyers' valuations has no effect on the expected revenue generated by a revenue-maximizing mechanism. This is despite the fact that the seller can create correlation in types. In more general private-value or interdependent-value environments, however, information disclosure matters. There, we establish that, in general, the best that the seller can do is to disclose no information at all.

Our findings extend in a straightforward manner to a number of other mechanism-design problems. If the interest is in designing efficient, instead of revenue-maximizing, mechanisms, then the previous analysis can be very easily modified. All that one needs to do, is to replace virtual surpluses with actual surpluses. For mechanism-design problems with multidimensional types, finding the optimal information-disclosure policy is not harder than finding the optimal disclosure policy for single dimensional types. All the steps described in the proof of Proposition 4 go through in this case, as well. However, solving the analog of Program $S_{G}$ in a multidimensional setting can be extremely difficult.

Suppose as we have so far, that the information that the seller observes about the buyers is statistically independent across them, but now, the ex-ante information of the buyers is statistically correlated. Then, when the conditions of Cremer and McLean (1988), or of McAfee and Reny (1992) are satisfied, the seller can extract full surplus, exactly as in those papers, so there is no further scope for information disclosure. If types consist of a payoff-relevant part, and a belief-relevant part, as in the work of Neeman (2004), and Heifetz and Neeman (2006), our results go through in the following sense. The correlation in the prior information can be exploited using "CremerMcLean" type of lotteries, to infer the buyers' valuations. When beliefs are part of types, however, the inference will typically not be perfect, so instead of learning the buyer's valuations, as in CM, the seller will have a sharper estimate of where they lie. After that initial step, we are essentially back to the case of statistically independent private information, with the priors replaced by the posteriors.

Finally, for the cases where the buyers' prior information is statistically independent, but the seller observes information that is statistically correlated across buyers, we conjecture that the seller will be able to use this information to her advantage, even in the baseline IPV case and, of course, in the case of interdependent values. The reason is that, there, the release of information can create correlation in the payoff-relevant part of the buyers' types, thus enabling the seller to use "Cremer-McLean" type of lotteries to extract more surplus from the buyers.

The main message of our analysis is that for a seller who has the power to choose the rules of trade based on the information that she has, sophisticated disclosure policies do not pay. Simply revealing nothing is optimal. In some special cases such as the classical independent private value paradigm, our information irrelevance result allows us to conclude that anything between no and full information disclosure is optimal. It would be interesting to investigate these questions within the framework of particular auction mechanisms. We leave this for future work. 


\section{Appendix A: Omitted Proofs}

\section{Proof of Proposition 4}

Take a disclosure policy $c$ and let $p(v, m, x)$ and $x(v, m, x)$ denote an optimal mechanism, given this disclosure policy. We show that if this mechanism is incentive-compatible, given $c$, it is also incentive-compatible when the seller discloses no information at all. If $p(v, m, x)$ and $x(v, m, x)$ is incentive-compatible, given $c$, then it must be the case that

$$
P_{i}\left(v_{i}, m_{i}, s_{i}\right)=E_{v_{-i}, s_{-i}, m_{-i}}\left[\Sigma_{z \in Z} p_{z}(v, s, m) \frac{\partial u_{i}^{z}\left(v_{i}, v_{-i}\right)}{\partial v_{i}} \mid m_{i}, s_{i}\right]
$$

is increasing in $v_{i}$ for each $m_{i}, s_{i}$.

When the seller discloses no information, then $P_{i}\left(v_{i}, s_{i}\right)=E_{v_{-i}, s_{-i}}\left[\Sigma_{z \in Z} p_{z}(v, s) \frac{\partial u_{i}^{z}\left(v_{i}, v_{-i}\right)}{\partial v_{i}} \mid s_{i}\right]$. We now show that irrespective of the information disclosure policy, it holds that

$$
P_{i}\left(v_{i}, s_{i}\right)=\Sigma_{m_{i}} \operatorname{prob}\left(m_{i} \mid s_{i}\right) P\left(v_{i}, m_{i}, s_{i}\right) .
$$

Observe that $P_{i}\left(v_{i}, s_{i}\right)$, and $P_{i}\left(v_{i}, m_{i}, s_{i}\right)$ can be written, respectively, as

$$
P_{i}\left(v_{i}, s_{i}\right)=\Sigma_{s_{-i} \in S_{-i}} \pi\left(s_{-i}\right) \int_{V_{-i}\left(s_{-i}\right)} \Sigma_{z \in Z} p_{z}(v, s) \frac{\partial u_{i}^{z}\left(v_{i}, v_{-i}\right)}{\partial v_{i}} f_{-i}\left(v_{-i} \mid s_{-i}\right) d v_{-i}
$$

and

$P_{i}\left(v_{i}, m_{i}, s_{i}\right)=\Sigma_{m_{-i} \in \mathcal{M}_{-i}} \Sigma_{s_{-i} \in S_{-i}} \int_{V_{-i}\left(s_{-i}\right)} \Sigma_{z \in Z} p_{z}(v, s, m) \frac{\partial u_{i}^{z}(v)}{\partial v_{i}} \frac{f_{-i}\left(v_{-i} \mid s_{-i}\right) \pi\left(s_{i}\right) \pi\left(s_{-i}\right) c(m \mid s)}{\pi\left(s_{i}\right) \Sigma_{s_{-i}} \pi_{-i}\left(s_{-i}\right) \Sigma_{m_{-i}} c(m \mid s)} d v_{-i}$.

Define $p_{z}(v, s)=\Sigma_{m \in \mathcal{M}} p_{z}(v, s, m) c(m \mid s)$. Then, (25) follows from the following considerations:

$$
\begin{aligned}
& \Sigma_{m_{i}} \operatorname{prob}\left(m_{i} \mid s_{i}\right) \cdot P\left(v_{i}, m_{i}, s_{i}\right) \\
= & \Sigma_{m_{i}}\left[\Sigma_{s_{-i}} \pi_{-i}\left(s_{-i}\right) \Sigma_{m_{-i}} c_{i}(m \mid s)\right] \cdot \Sigma_{m_{-i}} \Sigma_{s_{-i}} \int_{V_{-i}\left(s_{-i}\right)} \Sigma_{z \in Z} p_{z}(v, s, m) \frac{\partial u_{i}^{z}(v)}{\partial v_{i}} \frac{f_{-i}\left(v_{-i} \mid s_{-i}\right) \pi_{-i}\left(s_{-i}\right) c(m \mid s)}{\Sigma_{s_{-i}} \pi_{-i}\left(s_{-i}\right) \Sigma_{m_{-i}} c(m \mid s)} d v_{-} \\
= & \Sigma_{m_{i}} \Sigma_{m_{-i} \in \mathcal{M}_{-i}} \Sigma_{s_{-i} \in S_{-i}} \int_{V_{-i}\left(s_{-i}\right)} \pi_{-i}\left(s_{-i}\right) c(m \mid s) \Sigma_{z \in Z} p_{z}(v, s, m) \frac{\partial u_{i}^{z}(v)}{\partial v_{i}} f_{-i}\left(v_{-i} \mid s_{-i}\right) d v_{-i} \\
= & \Sigma_{s_{-i} \in S_{-i}} \pi_{-i}\left(s_{-i}\right) \int_{V_{-i}\left(s_{-i}\right)} \Sigma_{z \in Z} \Sigma_{m \in \mathcal{M}} p_{z}(v, s, m) c(m \mid s) \frac{\partial u_{i}^{z}(v)}{\partial v_{i}} f_{-i}\left(v_{-i} \mid s_{-i}\right) d v_{-i} \\
= & \Sigma_{s_{-i} \in S_{-i}} \pi_{-i}\left(s_{-i}\right) \int_{V_{-i}\left(s_{-i}\right)} \Sigma_{z \in Z} p_{z}(v, s) \frac{\partial u_{i}^{z}(v)}{\partial v_{i}} f_{-i}\left(v_{-i} \mid s_{-i}\right) d v_{-i} \\
= & P_{i}\left(v_{i}, s_{i}\right) .
\end{aligned}
$$

Now, from (24), we know that $P\left(v_{i}, m_{i}, s_{i}\right)$ is increasing in $v_{i}$ for each $m_{i}$, and $s_{i}$. Then, from (25), we can immediately conclude that $P_{i}\left(v_{i}, s_{i}\right)$ is increasing in $v_{i}$ for each $s_{i}$, which implies that the given mechanism is also incentive-compatible for the no information disclosure policy. 


\section{Appendix B: An Example where the Value of Program $S_{G}$ Depends on the Disclosure Policy}

Below, we describe an example that belongs in the private-values class and where the seller's revenue at the optimal mechanism (as well as the optimal mechanism) depends on the disclosure policy that she employs. This example illustrates how the no disclosure policy increases the seller's revenue by relaxing the incentive constraints. Its solution uses the technique described in Figueroa and Skreta (2007b). The reader interested in the full details behind the procedure used here is referred to that paper. ${ }^{46}$

There are two buyers, 1 and 2, and two allocations, $z_{A}$ and $z_{B}$. Buyer 1 's valuation, $v_{1}$, is distributed uniformly on the interval $[0,1]$, and his payoffs from these two allocations are given by, respectively:

$$
u_{1}^{z_{A}}\left(v_{1}\right)=v_{1}^{2}+0.37, u_{1}^{z_{B}}\left(v_{1}\right)=v_{1}^{5}+0.2 \text { for } v_{1} \in[0,1],
$$

which are both convex and increasing in $v_{1}$.

Buyer 2's valuation, $v_{2}$, is either 0 or 0.5 with equal probability, and his payoffs from allocations $z_{A}$ and $z_{B}$ are given by, respectively:

$$
\begin{aligned}
u_{2}^{z_{A}}(0) & =0 \text { and } u_{2}^{z_{B}}(0)=0 \\
u_{2}^{z_{A}}(0.5) & =0.2 \text { and } u_{2}^{z_{B}}(0.5)=0 .
\end{aligned}
$$

As can be readily verified from the buyers' payoff functions, this example falls within the privatevalue class, since no buyer's payoff depends on the valuation parameter of the other buyer.

The virtual utilities of allocations $z_{A}$ and $z_{B}$ are given by, respectively:

$$
\begin{aligned}
J_{z_{A}}\left(v_{1}, 0\right) & =3 v_{1}^{2}-2 v_{1}+0.37 \text { and } J_{z_{B}}\left(v_{1}, 0\right)=6 v_{1}^{5}-5 v_{1}^{4}+0.2 \\
J_{z_{A}}\left(v_{1}, 0.5\right) & =3 v_{1}^{2}-2 v_{1}+0.57 \text { and } J_{z_{B}}\left(v_{1}, 0.5\right)=6 v_{1}^{5}-5 v_{1}^{4}+0.2 .
\end{aligned}
$$

The seller observes a signal perfectly correlated with buyer 2's valuation, in other words, the seller learns 2's valuation. We consider the design of optimal auctions under two alternative disclosure policies: full and no information disclosure.

\section{Case 1: Optimal Mechanism under Full Disclosure (FD)}

The solution under full disclosure is as follows. When $v_{2}=0.5$, the seller assigns probability one to allocation $z_{A}$, irrespective of the value of $v_{1}$. This is because, as can be easily verified from

\footnotetext{
${ }^{46}$ Also, a fairly detailed account of the missing steps of the solution for this example, can be downloaded from the author's website.
} 
Figure 2, the virtual surplus of allocation $z_{A}$ is greater than the one of allocation $z_{B}$ for all values of $v_{1}$.

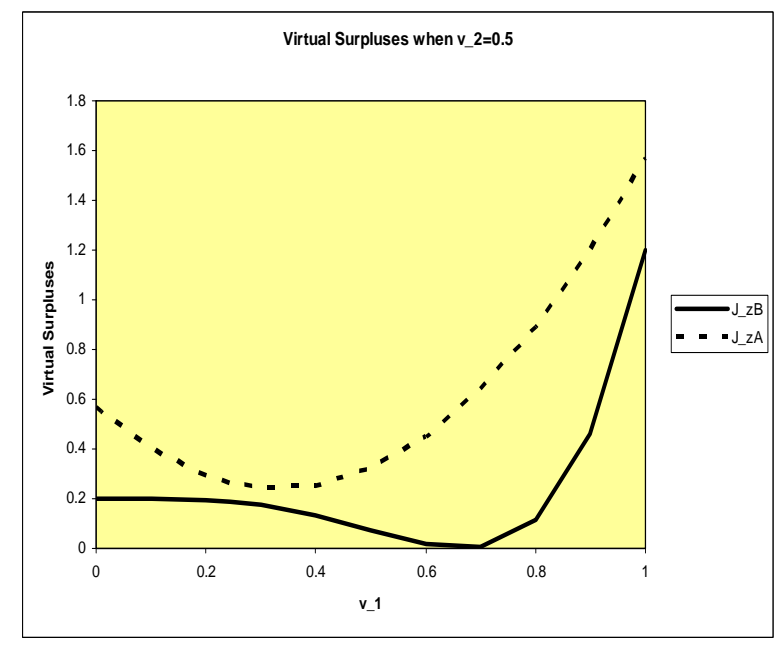

Figure 2

This assignment rule is feasible because when the seller assigns probability one to $z_{A}$, then (21) for buyer 1 reduces to

$$
P_{1}\left(v_{1}, 0.5,0.5\right)=\frac{d u_{1}^{z_{A}}\left(v_{1}\right)}{d v_{1}} \equiv P^{z_{A}}\left(v_{1}\right)=2 v_{1},
$$

which is increasing in $v_{1}$.

When $v_{2}=0$, the solution is derived as follows. First, let us the depict the virtual surpluses of allocations $z_{A}$ and $z_{B}$ :

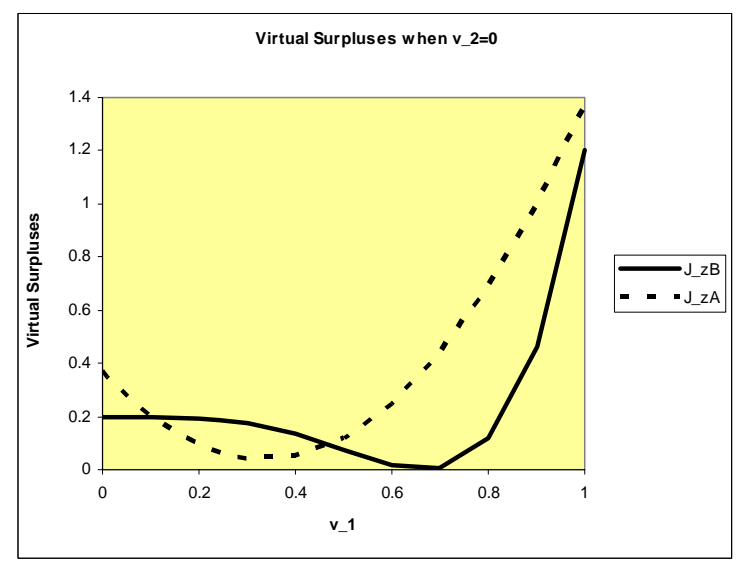

Figure 3 
The crossings of $J_{z_{A}}$ and $J_{z_{B}}$ occur at $\tilde{v}_{1}=0.10032$ and $\hat{v}_{1}=0.47064$.

When $v_{2}=0$ pointwise optimization of ${ }^{47}$

$$
\max _{(p, x)} \int_{[0,1]}\left[p_{z_{A}}\left(v_{1}, 0\right) J_{z_{A}}\left(v_{1}, 0\right)+p_{z_{B}}\left(v_{1}, 0\right) J_{z_{B}}\left(v_{1}, 0\right)\right] d v
$$

does not lead to a feasible mechanism. As we can see from Figure 3, pointwise optimization dictates to assign probability one to allocation $z_{A}$ for $v<0.10032$ and to assign probability one to allocation $z_{B}$ for $v$ in the interval [0.10032, 0.47064]. However, it is not possible to switch from allocation $z_{A}$ to allocation $z_{B}$ because this would imply that $P_{1}$ falls at 0.10032 since, as can be seen in Figure $4, P^{z_{A}}(v)>P^{z_{B}}(v)$ for all $v \in(0,0.737]$, where $P^{z_{A}}\left(v_{1}\right)=\frac{d u_{1}^{z_{A}}\left(v_{1}\right)}{d v_{1}}=2 v_{1}$, and $P^{z_{B}}\left(v_{1}\right)=\frac{d u_{1}^{z_{B}}\left(v_{1}\right)}{d v_{1}}=5 v_{1}^{4}$.

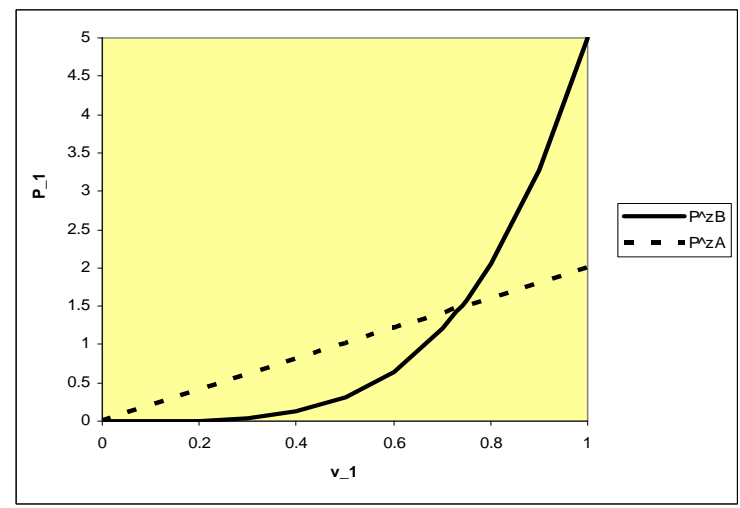

Figure 4

Following, the solution approach developed in Figueroa and Skreta (2007b), we search for the best region of compromise $[\underline{x}, \bar{x}]$ where the seller randomizes between $z_{A}$ and $z_{B}$ in such a way so as to minimize the loss from assigning positive weight to a sub-optimal allocation, while preserving incentive compatibility.

In the case of full disclosure, we find that the region of compromise is

$$
\left[\underline{x}^{F D}, \bar{x}^{F D}\right]=[0.0252,0.3169]
$$

\footnotetext{
${ }^{47}$ In what follows, we suppress the arguments $s$ and $m$ from the assignment rule, as they can be readily understood from the context, given the simplicity of the disclosure policies that we consider in this example.
} 
and the optimal assignment rule is:

$$
\begin{aligned}
& p_{F D}^{*}\left(v_{1}, 0\right)=(1,0) \text { for } v_{1} \in[0,0.0252) \\
& p_{F D}^{*}\left(v_{1}, 0\right)=\left(\frac{0.0504-5 v_{1}^{4}}{2 v-5 v_{1}^{4}}, 1-\frac{0.0504-5 v_{1}^{4}}{2 v-5 v_{1}^{4}}\right) \text { for } v_{1} \in[0.0252,0.3169) \\
& p_{F D}^{*}\left(v_{1}, 0\right)=(0,1) \text { for } v_{1} \in[0.3169,0.47064] \\
& p_{F D}^{*}\left(v_{1}, 0\right)=(1,0) \text { for } v_{1} \in(0.47064,1],
\end{aligned}
$$

which is incentive-compatible by construction. The seller's expected revenue in the case of full disclosure is

$$
\text { Revenue }_{F D}=0.39396 .
$$

\section{Case 2: Optimal Mechanism under No Disclosure}

In this case, buyer 1 does not know whether $v_{2}=0$ or $v_{2}=0.5$.

When $v_{2}=0.5$, the optimal assignment is exactly as in the case of full disclosure - namely, the seller assigns probability one to allocation $z_{A}$, and it is feasible for exactly the same reasons as before.

When $v_{2}=0$, as before, we are looking for a region $[\underline{x}, \bar{x}]$ where the seller sacrifices revenue by assigning positive weight also to the allocation that does not have the highest virtual valuation.

The only difference from the case of full disclosure is that, now, buyer 1 does not know $v_{2}$, so he is not sure what the optimal assignment is as a function of his report. This uncertainty relaxes the incentive compatibility constraints. For the case of no information disclosure, the region of compromise is

$$
\left[\underline{x}^{N D}, \bar{x}^{N D}\right]=[0.07,0.1391],
$$

which is smaller than the region of compromise under full disclosure described in (26).

The optimal assignment rule for this case is given by:

$$
\begin{aligned}
& p_{N D}^{*}\left(v_{1}, 0\right)=(1,0) \text { for } v_{1} \in[0,0.07) \\
& p_{N D}^{*}\left(v_{1}, 0\right)=\left(\frac{0.28-2 v_{1}-5 v_{1}^{4}}{\left(2 v_{1}-5 v_{1}^{4}\right)}, 1-\frac{0.28-2 v_{1}-5 v_{1}^{4}}{\left(2 v_{1}-5 v_{1}^{4}\right)}\right) \text { for } v_{1} \in[0.07,0.1391) \\
& p_{N D}^{*}\left(v_{1}, 0\right)=(0,1) \text { for } v_{1} \in[0.1391,0.47064] \\
& p_{N D}^{*}\left(v_{1}, 0\right)=(1,0) \text { for } v_{1} \in(0.47064,1],
\end{aligned}
$$

which is incentive-compatible by construction. The seller's expected revenue in the case of no information disclosure is

$$
\text { Revenue }_{N D}=0.40251 \text {. }
$$

Comparing the seller's revenue under full and no information disclosure, we see that

$$
\text { Revenue }_{N D}>\text { Revenue }_{F D} \text {. }
$$

Hence, we conclude that in this private-value setup information-disclosure policies affect the revenue generated by the optimal mechanism. 


\section{REFERENCES}

[1] Akerlof, G. (1970): "The Market for Lemons: Qualitative Uncertainty and the Market Mechanism." The Quarterly Journal of Economics 84(3):488-500.

[2] Bergemann, D., and M. Pesendorfer (2007): "Information Structures in Optimal Auctions," Journal of Economic Theory, 127, 580-609.

[3] Bergemann, D. and J. Valimaki (2006): "Information in Mechanism Design." In Richard Blundell, Whitney Newey and Torsten Persson, eds., Proceedings of the 9th World Congress of the Econometric Society. Cambridge: Cambridge University Press, 186-221.

[4] BoArd, S. (2006): "Revealing Information in Auctions: The Allocation Effect," Economic Theory, forthcoming.

[5] Bulow, J. And P. Klemperer (1996): "Auctions vs. Negotiations," American Economic Review, 86, No. 1 (March 1996):180-194.

[6] Cai H., J. Riley, And Yi L. (2007): "Reserve Price Signaling," Journal of Economic Theory, $135,253-268$.

[7] Cella, M. (2007): "Informed Principal with Correlation," mimeo University of Milan.

[8] Cremer, J., and R. McLean (1988): "Full Extraction of the Surplus in Bayesian and Dominant Strategy Auctions," Econometrica, 56, 1247-1258.

[9] Eso, P., And B. Szentes (2007): "Optimal Information Disclosure in Auctions and the Handicap Auction," Review of Economic Studies, 74, 705-731.

[10] Figueroa, N. And V. Skreta (2007): "The Role of Outside Options in Auction Design," mimeo.

[11] Figueroa, N. And V. Skreta (2007b): "A Note on Optimal Allocation Mechanisms," mimeo.

[12] Ganuza, J-J. (2004): "Ignorance Promotes Competition: An Auction Model with Endogenous Private Valuations," Rand Journal of Economics, 35, 583-598.

[13] Ganuza, J-J. and J.S. Penalva (2006): "On Information and Competition in Private Value Auctions," mimeo.

[14] Hansen, R. G. (2001): “Auctions of Companies," Economic Inquiry, Vol 39, 1, 30-43.

[15] Harstad, R. and V. Mares (2003): "Private Information Revelation in Common-Value Auctions," Journal of Economic Theory, 109, 264-282 
[16] Heifetz, A. and Z. Neeman, (2006): "On the Generic (Im)Possibility of Full Surplus Extraction in Mechanism Design," Econometrica, 2006, 74, 213-233.

[17] Jullien, B. And T. Mariotti, (2006): "Auction and the Informed Seller Problem," Games and Economic Behavior, 56, 2, 225-258.

[18] Kaplan, T.R. And S. Zamir (2002): "The Strategic Use of Seller Information in PrivateValue Auctions," mimeo.

[19] Kaplan, T.R. and S. ZamiR (2007): "Asymmetric First-Price Auctions with Uniform Distributions: Analytic Solutions to the General Case," mimeo.

[20] Kremer, I. and Skrzypacz, A. (2004): "Auction Selection by an Informed Seller," mimeo.

[21] Landsberger, M., Rubinstein, J., Zamir S. and E. Wolfstetter (2001): "First Price Auctions When the Ranking of Valuations is Common Knowledge," Review of Economic Design $6,461-480$.

[22] Lebrun, B. (1999): "First Price Auctions in the Asymmetric N Bidder Case," International Economic Review, 40, 125-142.

[23] Maskin, E. And J. Tirole, (1990): "The Principal-Agent Relationship with an Informed Principal: The Case of Private Values," Econometrica, 58, 379-409.

[24] Maskin, E. and J. Tirole (1992): "The Principal-Agent Relationship with an Informed Principal, II: Common Values," Econometrica, 60: 1-42.

[25] McAfee P. And P. Reny, (1992): "Correlated Information and Mechanism Design," Econometrica, 60, 395-421.

[26] Milgrom, P. (2004): "Putting Auction Theory to Work," Cambridge University Press.

[27] Milgrom, P. and I. Segal, (2002): "Envelope Theorems for Arbitrary Choice Sets," Econometrica, 70, 583-601.

[28] Milgrom, P. And R.Weber (1982): "A Theory of Auctions and Competitive Bidding", Econometrica, 50, 1089-1122.

[29] Mookherjee, D. And S. Reichelstein, (1992): "Dominant Strategy Implementation of Bayesian Incentive Compatible Allocation Rules," Journal of Economic Theory, 56, 378-399.

[30] Myerson, R. (1979): "Incentive Compatibility and the Bargaining Problem," Econometrica 47, 61-73.

[31] Myerson, R. (1981): "Optimal Auction Design," Mathematics of Operations Research 6, 58-73. 
[32] Myerson, R. (1983): "Mechanism Design by an Informed Principal," Econometrica 51, 17671798.

[33] Myerson, R. (1986): "Multistage Games with Communication." Econometrica 54, 323- 358.

[34] Mylovanov, T. (2005): "The Informed Principal Problem with Independent Private Values and Quasilinear Preferences," mimeo University of Bonn.

[35] Neeman, Z. (2004): "The Relevance of Private Information in Mechanism Design," Journal of Economic Theory, 117, 55-77.

[36] Obara, I. (2008): "The Full Surplus Extraction Theorem with Hidden Actions," Advances in Theoretical Economics, forthcoming.

[37] Ottaviani, M. and A. Prat,(2001): “The Value of Public Information in Monopoly," Econometrica, 69, 1673-1683.

[38] Perry, M., and P. Reny (1999): "On the Failure of the Linkage Principle in Multi-Unit Auctions," Econometrica, 67, 895-900.

[39] Riley, J. G. And W. F. Samuelson (1981): "Optimal Auctions," American Economic Review, 71, 381-392.

[40] Skreta, V. (2006): "Mechanism design for arbitrary type spaces," Economics Letters, 91, 293-299.

[41] Skreta, V. (2007): "Optimal Auctions with General Distributions," mimeo.

[42] Skreta, V. (2007b): "Auction Design by an Informed Seller: Interdependent Values," in progress.

[43] TislJar, R. (2002): "Mechanism Design by an Informed Principal - Pure-Strategy Equilibria for a Common Value Model," mimeo University of Bonn.

[44] Tisluar, R. (2003): "Optimal Trading Mechanisms for an Informed Seller," Economics Letters, $81,1-8$.

[45] Yilankaya, O. (1999): “A Note on the Seller's Optimal Mechanism in Bilateral Trade with two-sided Incomplete Information," Journal of Economic Theory 87, 125-143. 